\title{
Traumatic Brain Injury as a Disorder of Brain Connectivity
}

\author{
Jasmeet P. Hayes, ${ }^{1,2,3}$ Erin D. Bigler, ${ }^{4,5,6}$ AND Mieke Verfaellie ${ }^{2,7}$ \\ ${ }^{1}$ National Center for PTSD, VA Boston Healthcare System, Boston, Massachusetts \\ ${ }^{2}$ Department of Psychiatry, Boston University School of Medicine, Boston, Massachusetts \\ ${ }^{3}$ Neuroimaging Research for Veterans Center, VA Boston Healthcare System, Boston, Massachusetts \\ ${ }^{4}$ Department of Psychology, Brigham Young University, Provo, Utah \\ ${ }^{5}$ Neuroscience Center, Brigham Young University, Provo, Utah \\ ${ }^{6}$ Department of Psychiatry, University of Utah, Salt Lake City, Utah \\ ${ }^{7}$ Memory Disorders Research Center, VA Boston Healthcare System, Boston, Massachusetts
}

(Received April 3, 2015; Final Revision August 4, 2015; Accepted August 11, 2015)

\begin{abstract}
Objectives: Recent advances in neuroimaging methodologies sensitive to axonal injury have made it possible to assess in vivo the extent of traumatic brain injury (TBI) -related disruption in neural structures and their connections. The objective of this paper is to review studies examining connectivity in TBI with an emphasis on structural and functional MRI methods that have proven to be valuable in uncovering neural abnormalities associated with this condition. Methods: We review studies that have examined white matter integrity in TBI of varying etiology and levels of severity, and consider how findings at different times post-injury may inform underlying mechanisms of post-injury progression and recovery. Moreover, in light of recent advances in neuroimaging methods to study the functional connectivity among brain regions that form integrated networks, we review TBI studies that use resting-state functional connectivity MRI methodology to examine neural networks disrupted by putative axonal injury. Results: The findings suggest that TBI is associated with altered structural and functional connectivity, characterized by decreased integrity of white matter pathways and imbalance and inefficiency of functional networks. These structural and functional alterations are often associated with neurocognitive dysfunction and poor functional outcomes. Conclusions: TBI has a negative impact on distributed brain networks that lead to behavioral disturbance. (JINS, 2016, 22, 120-137)
\end{abstract}

Keywords: Diffusion tensor imaging, white matter, fMRI, neural networks, corpus callosum, diffuse axonal injury

\section{INTRODUCTION}

One of the most important contributions of Norman Geschwind's seminal papers on disconnection syndrome (1965) was the idea that higher brain functions arise from distributed brain networks that, when lesioned, produce behavioral disturbance (Catani, 2005). Traumatic brain injury (TBI) provides a prime example of such network dysfunction (Bigler, 2001). The seminal post-mortem and histological studies by Strich (1956) and Peerless and Rewcastle (1967) established more than half a century ago that TBI may be characterized largely as a white matter disorder (see also reviews by Johnson et al., 2013; Smith et al., 2003). Axonal injury interferes with neural

Correspondence and reprint requests to: Jasmeet P. Hayes, National Center for PTSD (116B-2), VA Boston Healthcare System, $150 \mathrm{~S}$. Huntington Avenue, Boston MA 02130. E-mail: jphayes@bu.edu communication, and as such, disrupts the neural networks that link brain structure to function.

Neuropsychological outcome in TBI has long been assumed to relate to the degree to which TBI disrupts white matter and neural network functioning, as well as the ability of those networks to recover, adapt or reconstitute (Gale et al., 1995; Ruppin \& Reggia, 1995). However, until recently, there were no effective neuroimaging techniques that permitted direct evaluation of white matter pathways (see Corbetta, 2012; Sharp et al., 2014; Wilde et al., 2012), other than the gross identification of visibly obvious traumatic abnormalities (Bigler, 2001). Innovative neuroimaging methodologies in conjunction with neurocognitive and functional outcome studies of TBI in recent years have provided valuable knowledge of how changes in cerebral tracts relate to behavior and cognition following TBI.

The objective of this paper is to summarize research examining brain connectivity in TBI. We begin with a précis of the neuropathological correlates of TBI and discussion of 
the various neuroimaging methods that have been used to detect TBI in vivo and assess network functionality. We then review structural neuroimaging findings, with an emphasis on the implications of observed alterations for cognitive and functional outcomes. As an important complement to the structural findings, we describe findings from resting-state functional connectivity MRI studies of TBI to delineate a broader view of how integrated networks of gray and white matter are altered in TBI. We limit this review to studies of adult TBI, and refer the reader to recent reviews of the pediatric TBI literature (Ashwal et al., 2014; Suskauer \& Huisman, 2009; Wilde et al., 2012).

\section{PATHOPHYSIOLOGY OF TBI}

Axonal injury is the primary neuropathology associated with TBI. Depending on the severity of the TBI, axonal injury can manifest as microscopic diffuse injury or as macroscopic focal lesions superimposed on diffuse injury (Adams et al., 1989). Until recently, it was generally believed that the physical trauma sheared axons upon impact, stretching axons beyond tolerance and leading to axonal disconnection at the time of the injury. Although this does occur in more severe injuries, more recent work has made clear that TBI-related cell damage encompasses a variety of interrelated primary and secondary pathological events that occur progressively and lead to axonal disconnection over time, termed traumatic axonal injury (TAI; Povlishock \& Christman, 1995). When trauma induces structural changes to the axolemma, an influx of potassium and calcium ions occurs, leading to loss of ionic homeostasis and disruption of axonal transport (Bigler \& Maxwell, 2012). If the injury is mild enough, these neurometabolic changes may be reversible; however, these changes often progress to axonal swelling and secondary axotomy over the course of hours in animals (Povlishock et al., 1983) and days to months in humans (Blumbergs et al., 1995). The severity of axonal injury is often tied to the duration of loss of consciousness (LOC) and other markers of severity of injury. TAI can injure small clusters of axons in any given area (Taber \& Hurley, 2013) that may be difficult to visualize and detect, but nevertheless may profoundly disrupt behavior. This highlights the need for sensitive techniques to image white matter abnormalities. Furthermore, the diffuse nature of axonal injury highlights the potential impact on higher order functions that rely on the integrity of neural networks.

\section{IMAGING WHITE MATTER IN TBI}

Clinically, the most common imaging methods used to detect TBI-associated neuropathology include computed tomography (CT), fluid attenuated invasion recovery (FLAIR) MR, and T1-weighted MR sequences. CT and FLAIR sequences are most useful for identifying hemorrhagic bleeds and other macroscopic intracranial injuries. Traumatic hemorrhage often leaves the blood byproduct hemosiderin, which shows up as a hypointense (dark) signal on MRI studies, especially on T2-and susceptibility weighted images. T1-weighted images, which approximate gross anatomy, provide basic white and gray matter differentiation sufficient for quantification of brain volume (Bigler et al., 2010).

Although these imaging techniques have allowed detection of gross white matter pathology associated with TBI, they are limited in that they are unable to locate specific pathways within the white matter or to assess pathway integrity. Furthermore, most TBIs are non-hemorrhagic, and thus undetectable on some of the most commonly used clinical scans. As a result, the extent of injury to the brain may be underestimated. The insensitivity of conventional imaging methods may have spurred the idea that TBI, particularly within the mild range, is not associated with chronic structural damage. This changed with the advent of MR diffusion tensor imaging (DTI), which has made the detection of white matter microstructure alterations possible (Hulkower et al., 2013; Shenton et al., 2012).

\section{Diffusion Tensor Imaging}

DTI is a non-invasive imaging technique sensitive to the movement of water molecules in nervous tissue (Jones, 2008). When unrestricted, water diffuses randomly, whereas it is hindered in its movement in highly organized tissue such as collections of nerve fibers composing white matter. As illustrated in Figure 1, the combination of axonal membrane and myelin coating form barriers restricting intra-axonal water dispersion (vertically oriented lines in the diagram). The flow and dispersion of water molecules are in a direction mostly perpendicular to the confining membrane wall. If degradation from trauma occurs within the axonal membrane and surrounding myelin, water dispersion is less restricted. When the level of damage in TBI reaches a threshold where the membrane becomes leaky, breaks down, or is literally sheared away, DTI detects the signal changes in water dispersion that reflect breaches in white matter integrity.

Some of the most frequently used DTI metrics useful in quantifying pathological change in white matter include the apparent diffusion coefficient (ADC) and fractional anisotropy (FA). Other often-used metrics are mean diffusivity (MD), axial diffusivity (AxD), and radial diffusivity (RD). ADC and MD measure the extent to which water is impeded in the tissue of interest. Pathologically high ADC or MD values may reflect unimpeded motion and are suggestive of decreased integrity of neuronal structure, whereas low values are thought to reflect cellular edema and inflammation. DTI involves the use of a tensor model to extract the directionality of water flow in axonal fiber tracts. FA, perhaps the most frequently used index in DTI studies, measures the extent to which water displacement is directionally dependent in its flow along the cell. High FA values are indicative of strong anisotropy, putatively associated with intact white matter microstructure. However, elevated FA beyond an expected normative value may be a sign of inflammation, where perturbation of white matter has the potential to stimulate an inflammatory response (Bramlett \& Dietrich, in press). AxD reflects water 
(a)

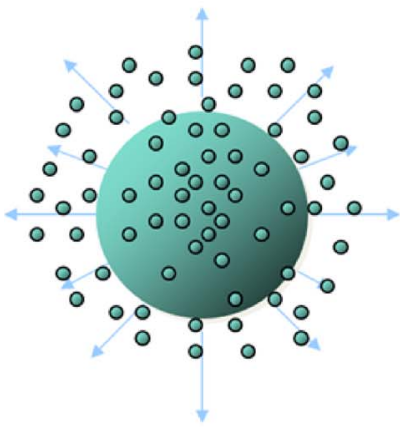

FA - low

ADC/MD - high (b)

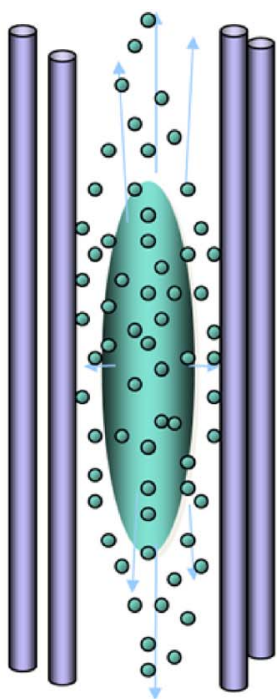

FA - high

ADC/MD - low (c)

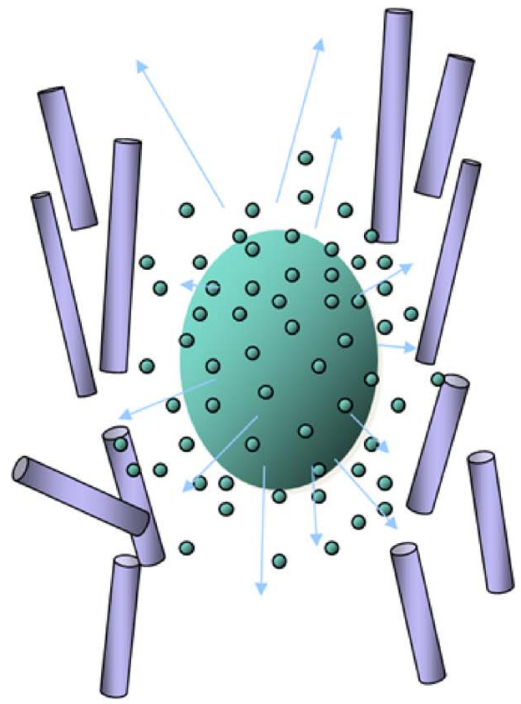

FA - decreased

ADC/MD - increased

Fig. 1. (a) This diagram depicts unconstrained molecular movement of a water droplet when there are no membranes to hold the water within. (b) Vertically oriented axon with a membrane wall that constrains the direction of movement of intracellular water as it moves in the same vertical direction (see up-and-down arrows) as the constraining membrane. However, as shown in (c), if the membrane breaks down or is degraded, water molecules disperse in a similar manner to the unconstrained condition. FA = fractional anisotropy, $\mathrm{ADC}=$ apparent diffusion coefficient, $\mathrm{MD}=$ mean diffusivity. Figure was created by Geri R. Hanten, Ph.D., Baylor College of Medicine.

diffusion along the longitudinal axis of the tensor and, although controversial, is purported to be a measure of axonal structure and damage. $\mathrm{RD}$ reflects diffusion along the two non-primary axes of the tensor and is purported to be a measure of myelin integrity.

Since similarly oriented white matter fibers will possess similar water diffusion properties, aggregated axons traveling together may be extracted (Choudhri et al., 2014), a method referred to as DTI tractography. This method represents a key neuroimaging tool for network identification (Catani \& Thiebaut de Schotten, 2008) and allows traumatic axonal injury in TBI to be visualized as an interruption or degradation of fibers within specific white matter tracts (see Figure 2).

Although DTI represents a great improvement in terms of sensitivity to traumatic axonal injury, there is currently no one accepted standard data analysis protocol, making cross-study comparisons challenging. DTI metrics can be derived from several methods including ROI-based methods, programs such as tract-based spatial statistics (TBSS) that allow for whole-brain analysis, and tractography. After data collection, DTI data are subjected to an array of processing steps before they are interpretable. In the pre-processing pipeline, decisions need to be made regarding how to deal with eddy current artifacts and subject motion, although improvements in imaging hardware over the last decade have reduced concerns of motion sensitivity and signal distortions for DTI data (Tournier et al., 2011). With regard to post-processing, there are different methods for estimating the tensor and deriving quantitative measures, which may influence the results (for a review, see Jones \& Cercignani, 2010). As such, consideration of the analytic methods used is important in interpreting findings.

\section{Comparison of Methods to Detect TBI}

Several studies have now shown that DTI methods can uncover white matter abnormalities not visible on conventional clinical scans (Huisman et al., 2004; Kim et al., 2013; Skoglund et al., 2008), suggesting that there are likely to be more significant structural changes following TBI than previously assumed. Furthermore, unlike conventional scans, diffusion methods such as tractography can visualize long-coursing connections that can potentially be affected by focal injury produced by the injury force. This is demonstrated in Figure 3, which illustrates different imaging modalities in a patient with severe TBI who suffered extensive frontal lobe damage as a result of surface and deep contusions. These are visualized on CT scan conducted on the day of injury (Figure 3a). MR studies performed more than 4 years later reflect the chronic pathological changes. The axial FLAIR MRI sequence clearly shows a white matter signal abnormality identified as a hyperintensity in the left frontal lobe (white arrow, Figure 3d). The white matter damage can also be seen in the T1 images, but not as distinctly as in the FLAIR image. There has also been loss of gray matter and the development of focal degeneration, referred to as encephalomalacia, in both the frontal and temporal polar areas (Figure 3b, 3c). Furthermore, as observed in the mid-sagittal T1 MRI, frontal white matter 
damage affected interhemispheric pathways across the anterior corpus callosum and reduced its size (Figure $3 \mathrm{e}, \mathrm{f}$ ). Returning to the FLAIR image, even though the left frontal lobe hyperintense white matter signal is so prominent and divergent from its contralateral counterpart, it would be a mistake to assume that this white matter damage and the surrounding focal encephalomalacia produces just a focal effect. This becomes evident when viewing the DTI tractography map (taken from a healthy control subject; Figure 3g), which shows six white matter pathways that would normally course through the site of focal frontal parenchyma damage sustained by the TBI patient and would conceivably be influenced by the injury. Thus, focal white matter damage has the potential to influence major fiber tracts, some of which may disrupt functioning distal to where the focal white matter damage occurred. Although not shown in the figure, it is important to note that damage to white matter tracts due to the force of the initial injury may independently contribute to loss in connectivity.

\section{FUNCTIONAL NETWORKS IN TBI}

Brain regions and their interconnecting white matter pathways form the anatomical building blocks of the brain, but brain function arises from the integrated functioning of neural networks as a whole. Resting state functional connectivity MRI (rs-fcMRI; see Smith, 2012; Zhang \& Li, 2012) is a relatively new method that permits the examination of intrinsic connectivity networks. It does so by assessing how "functionally connected" different regions are, based on examination of spontaneous fluctuations in blood oxygenation level-dependent (BOLD) signal. The assumption is that the more closely in-phase the activity in two regions is, the stronger their connectivity (Sporns, 2014; Yeo et al., 2011). Rs-fcMRI data are typically collected while the subject is "at rest" (i.e., lying still with eyes closed or looking at a fixation point), although spontaneous fluctuations in the BOLD signal can also be extracted from task-based fMRI studies. Furthermore, the integrity of intrinsic connectivity networks can also be assessed by examining task-evoked changes in

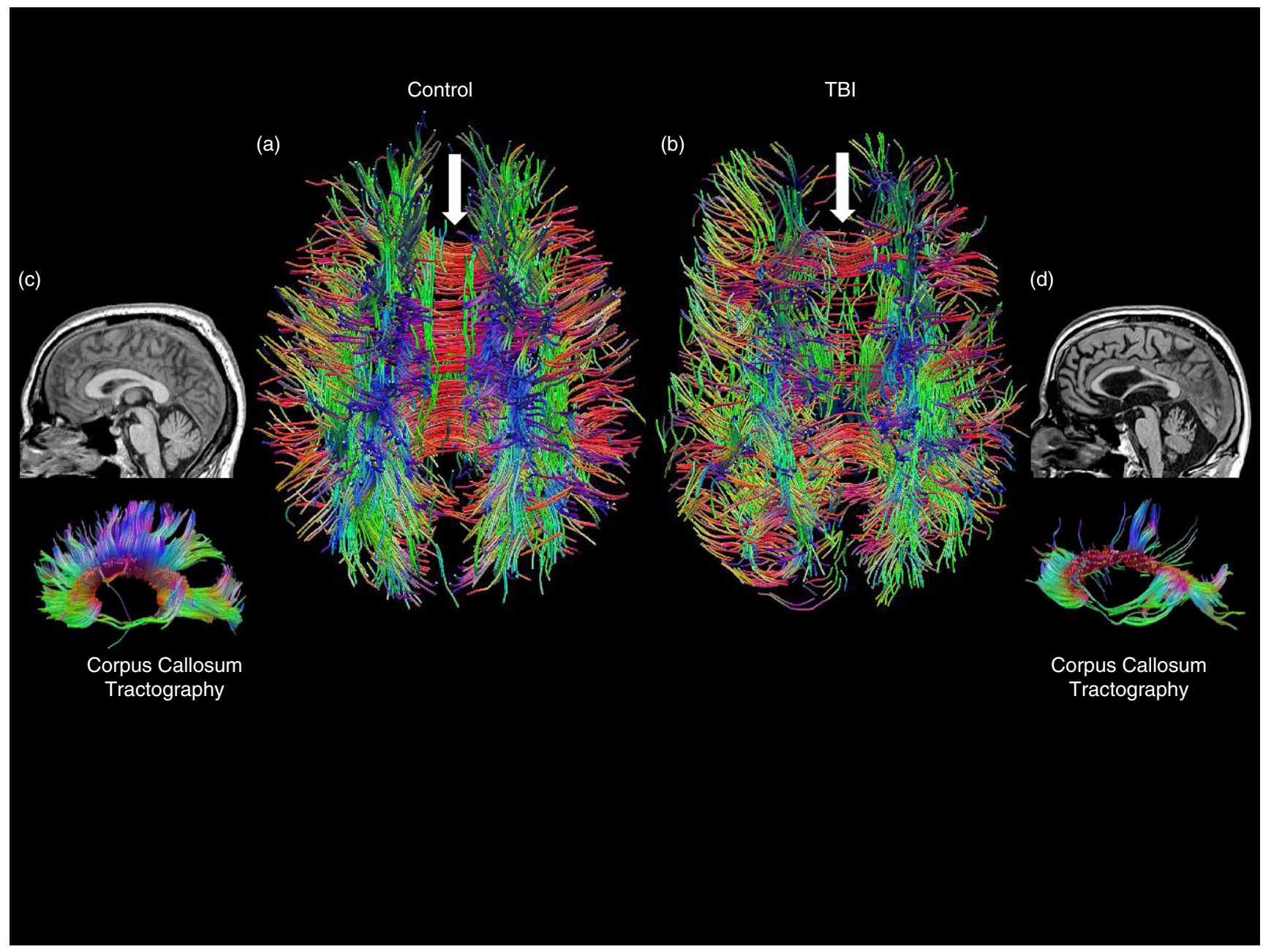

Fig. 2. (a) Dorsal view of an age-matched control whole-brain tractography image compared to (b) a patient with severe TBI. The white arrows point to the corpus callosum, which is intact in the control but with major tract loss in the TBI patient. Likewise, a mid-sagittal T1 MRI view shows an intact corpus callosum in the control subject (c) and a withered, atrophied one in the TBI patient (d). More dramatically, DTI tractography of the corpus callosum shows extensive loss of callosal tracks in the TBI patient compared to the control. 


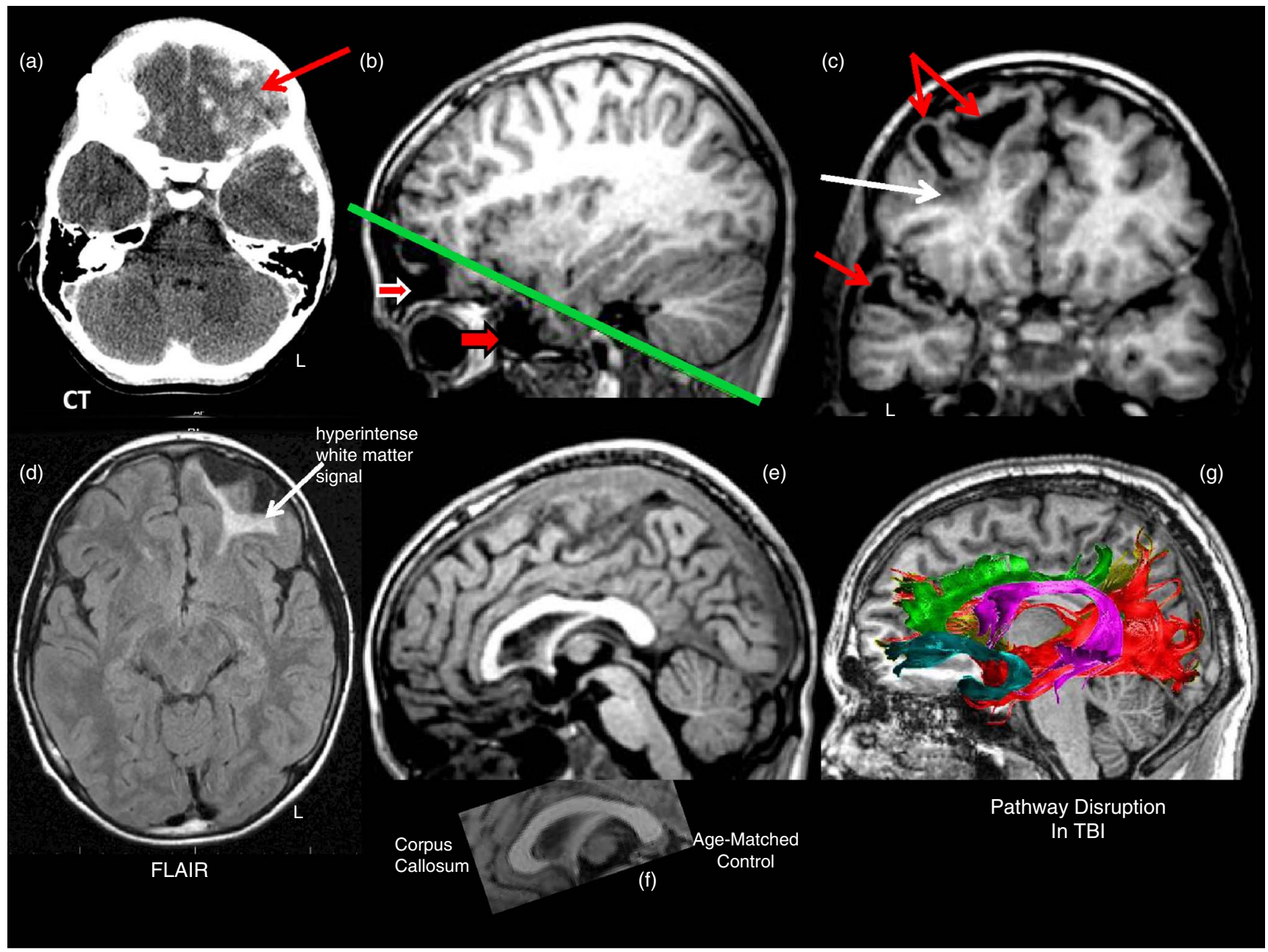

Fig. 3. (a) Day-of-injury computed tomogram (CT) showing extensive hemorrhagic lesions (white scattered lesions) especially notable in the inferior left frontal region (red arrow). (b) The sagittal T1-weighted MRI obtained four years post-injury shows extensive left anterior inferior frontal (top red arrow with white border) and temporal pole (bottom red arrow with dark border) wasting, referred to as encephalomalacia. The green line depicts the orientation of the oblique coronal cut as shown in (c). The particularly dark signal (white arrow) underlying the focal encephalomalacia represents hemosiderin. (d) In contrast, the MR signal in the white matter regions of the frontal and temporal lobes as depicted in the T1 images is abnormal, but not as distinct as the hyperintense signal in the axial fluid attenuated inversion recovery (FLAIR) sequence. T1 mid-sagittal MRI showing reduced size of the anterior aspect of the corpus callosum (e) in comparison to an age-matched control (f). (g) Pathways in the normal brain that would normally course through what is now damaged parenchyma and thus would be damaged in the TBI patient. These include, in addition to the corpus callosum, the anterior cingulum (green), occipitofrontal fasciculus (brown), uncinate fasciculus (teal), occipitotemporal fasciculus (red), and arcuate fasciculus (purple).

networks thought to mediate task performance. This approach allows for a more direct evaluation of the functional role of networks in specific aspects of cognition.

Yeo and colleagues (2011) have shown the existence of seven major intrinsic connectivity networks of the human cerebral cortex, which could be further parcellated into 17 smaller subnetworks. These include sensory and motor networks, as well as association networks involved in different aspects of cognition (see Figure 4). Importantly, while several of these association networks are activated by externally oriented tasks (i.e., task positive networks), the default mode network consists of a set of brain regions that show highly correlated brain activity during rest and a reduced level of connectivity during most attentionally demanding tasks. Within networks, some regions are identified as hubs because they play a key role in information transfer and integration within the network. Moreover, several hubs are densely interconnected, forming a structural core known as a "rich club" that may serve to link distinct functional networks and coordinate overall brain communication (van den Heuvel \& Sporns, 2011). An important implication of this network topology is that the impact of focal lesions as a result of TBI will depend not only on the size of the lesion, but also on the centrality of the nodes and pathways that it disrupts.

Rs-fcMRI networks such as the default mode network have been shown to be relatively robust and reliable, 


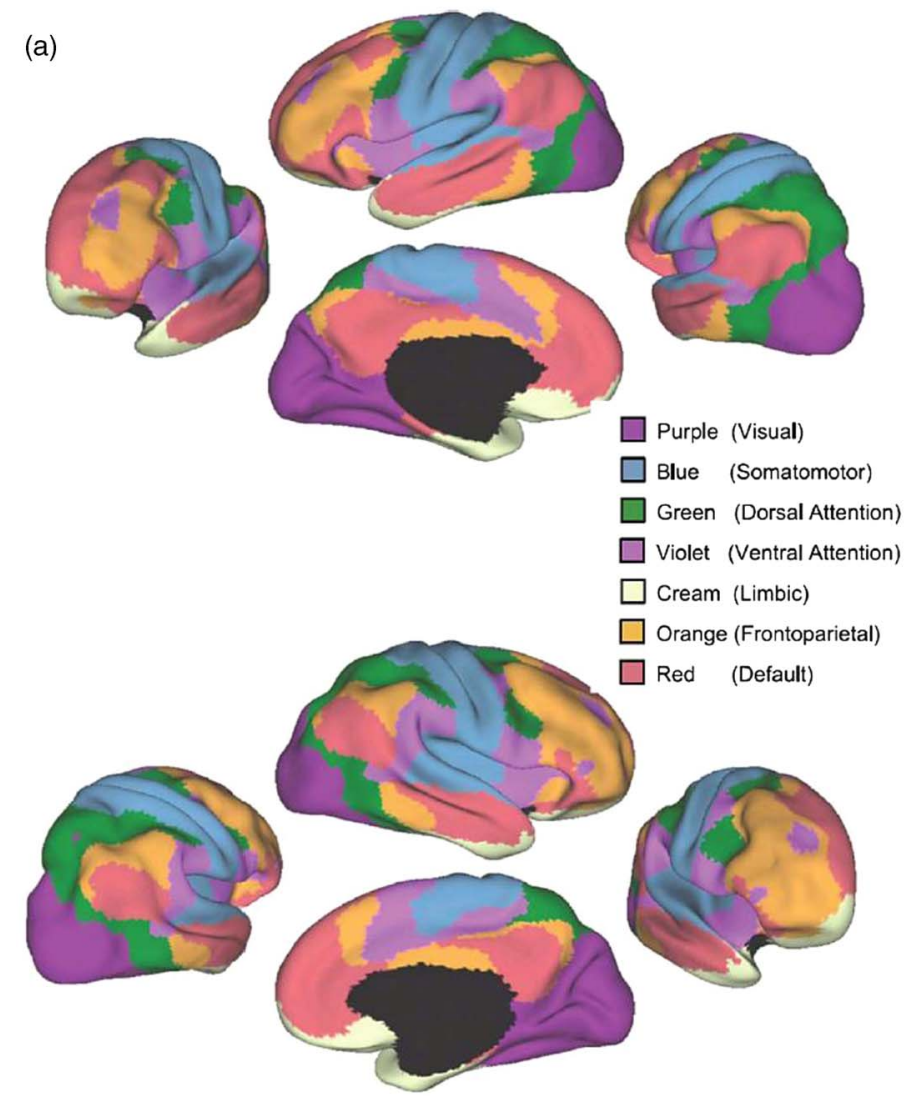

(b)
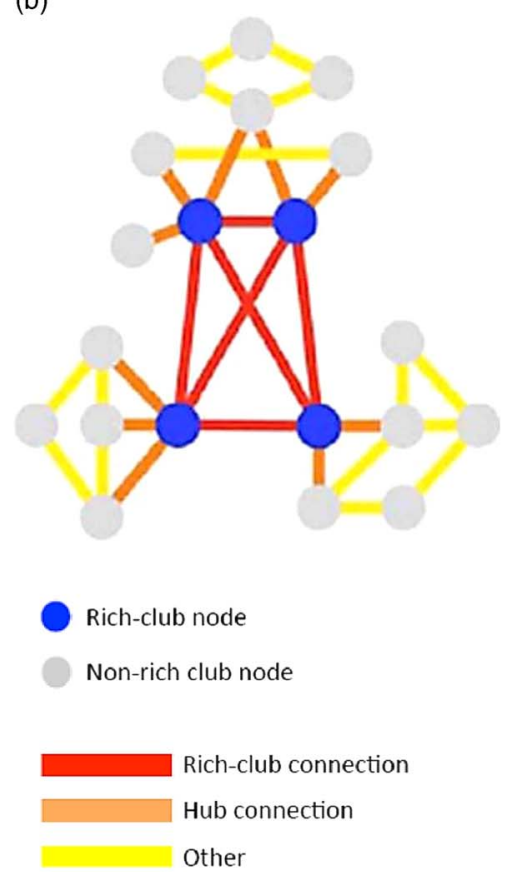

Fig. 4. (a) Using rs-fcMRI mapping, a seven-network parcellation of the human cerebral cortex is presented, derived from 1,000 subjects from Yeo and colleagues (2011) (used with permission from the American Physiological Society). (b) Using both rs-fcMRI and DTI, van den Heuvel and Sporns (2011) derived a rich club network. When rich-club networks are inlaid within other networks, the complexity of network integration can be visualized. An injury to rich-club networks (blue circles and red connections) is much more disruptive to network integrity than damage to non-rich-club nodes and connections (gray circles, and orange and yellow connections) because of the dense interconnectedness of rich-club networks. In this analogy, the amount of damage is the same but the effects would be widely different because of the location and how networks would be affected. Adapted with permission from the Society for Neuroscience.

particularly in comparison to results from task-based fMRI studies. However, as with DTI data, there are multiple approaches to rs-fcMRI data analysis and the selection of pre- and post-processing procedures can influence outcomes (see Cole et al., 2010). For instance, the results from seedbased connectivity analyses, which require a priori selection of a brain region, may vary based on the specific coordinates of the seed location. Independent components analysis, a commonly used data-driven approach, is not subject to the limitation of seed based analysis; however, this approach comes with its own difficulties as varying thresholds for component selection may impact comparability across studies. Other factors that influence the results include decision points in the pre-processing procedure, such as how head motion and confounding signals in the cerebrospinal fluid and white matter (e.g., due to scanner instabilities and respiration) are dealt with. Until greater consensus is reached regarding optimal methods, comparison across studies will remain a challenge, as will the application of these imaging methods in clinic settings.

\section{TBI-ASSOCIATED STRUCTURAL CONNECTIVITY ABNORMALITIES}

\section{Tracts Most Often Impacted by TBI}

Some of the tracts most vulnerable to head injury include long coursing and commissural fibers. Midline structures such as the corpus callosum may be more susceptible to rotational and shearing forces than other brain regions (Gentry et al., 1988; Reinarz et al., 1988; Tate \& Bigler, 2000). Consistent with this, a landmark port-mortem study reported damage to axons in the corpus callosum and fornix following TBI of varying severity, suggesting particular vulnerability in these regions to axonal injury (Blumbergs et al., 1995). DTI studies have largely supported this postmortem finding by providing in vivo evidence for the vulnerability of these midline regions (Kinnunen et al., 2011; Kraus et al., 2007). Other major tracts that have consistently shown abnormalities in TBI include the internal capsule (Kraus et al., 2007), superior and inferior longitudinal fasciculi (Kinnunen et al., 2011), and cerebellar 
fibers (Mac Donald et al., 2011; Warden et al., 2009). With increasing injury severity, involvement of the brainstem is highly likely, leading to transient loss of consciousness and coma. According to one influential model of TBI, injury to the brain occurs in a centripetal manner (Ommaya \& Gennarelli, 1974), with loss of consciousness resulting from greater disruptions between deep structures (e.g., brainstem) and cortical and subcortical structures.

\section{White Matter Disruption Observed in Cross-sectional Studies}

Alterations in white matter integrity are observed as early as within 24 hours of injury (Marmarou et al., 2006; Newcombe et al., 2007) and are still evident several years post-injury (Kennedy et al., 2009; Kraus et al., 2007; Nakayama, 2006; Palacios et al., 2012; Xu et al., 2007). Studies of chronic (i.e., $>3$ months post-injury) moderate to severe TBI reliably reveal decreased anisotropy and increased diffusivity, suggesting loss of axonal integrity and myelin degeneration. As an example, Kraus et al. (2007) demonstrated reductions in FA in several fiber tracts including the internal capsule, corpus callosum, and superior longitudinal fasciculus, among other regions, in adults with moderate to severe TBI. Consistent with these results, Palacios et al. (2012) found TBI-related FA reductions in the corpus callosum, superior longitudinal fasciculus, and other long and short fibers in participants 2 months to 21 years post-injury. Taken together, the findings suggest that white matter abnormalities following moderate to severe TBI persist in the chronic stage and while there is variability in the tracts that have been shown to be abnormal, some consistent findings also emerge.

Individuals with mild TBI (mTBI) also exhibit white matter abnormalities early in the course of injury recovery (Lo et al., 2009), and in a subset of individuals, these abnormalities persist years post-injury (Fakhran et al., 2013; Geary et al., 2010; Lipton et al., 2008). As in more severe injury, commissural and long coursing fibers are altered in mTBI, albeit less extensively so (Goetz et al., 2004; Kraus et al., 2007). However, there is less anatomical consistency in terms of injured regions in mTBI than in moderate and severe TBI, which may be related to mechanism and type of injury. Nonetheless, a recent metaanalysis of studies of mTBI concluded that the corpus callosum is particularly vulnerable (Aoki et al., 2012).

\section{Sports Concussion}

mTBI has been studied extensively in the context of sports concussion. A recent review of DTI studies of sports-related mTBI led to the conclusion that microstructural white matter abnormalities may be present both in the acute and in the sub-acute/chronic phase in mild injury (Gardner et al., 2012). Of particular concern is that athletes in sports such as boxing and football are often subjected to repetitive concussions and subconcussive hits that cumulatively impact brain structure and cognitive function. The adverse consequences of repetitive injury have gained greater attention recently due to reports of poor long-term neural and behavioral outcomes in professional football players. The few human studies that have systematically examined this issue suggest that white matter abnormalities increase with the number of repetitive concussions and subconcussive hits (Bazarian et al., 2012; Koerte, Ertl-Wagner, et al., 2012; Koerte, Kaufmann, et al., 2012). Additional studies are now underway to uncover the relationship between repetitive head injury and neurodegenerative disease such as chronic traumatic encephalopathy (McKee et al., 2013).

\section{Blast Exposure}

Another increasingly common cause of mTBI is blast exposure. The consequences of blast-induced brain injury have gained visibility in recent years in large part due to the stateside return of thousands of Iraq and Afghanistan service members exposed to explosive ordnance such as improvised explosive devices and rocket propelled grenades. One important difference between blast-induced mTBI and mTBI from civilian events is that the acceleration/deceleration effects that are typical of sports and motor vehicle trauma are less likely to occur following exposure to primary blast. Naturally, an important question that arises is whether distinct mechanisms (e.g., blast $v s$. direct head impact) result in different neural sequelae. Few studies have directly examined the impact of injury mechanism on white matter, but studies of primary blast injury show white matter abnormalities that appear to mimic the abnormalities seen in the civilian literature (Hayes et al., 2012; Warden et al., 2009). In actuality, the majority of blast injuries involve multiple mechanisms, both blast and acceleration/deceleration forces that arise from secondary and tertiary blast effects. Thus, the bulk of studies examining military blast mTBI have included individuals with both primary blast exposure and additional secondary and tertiary injuries. These studies have generally shown decreases in white matter integrity both in the subacute (Mac Donald et al., 2011) and chronic phase (Davenport et al., 2012; Hayes et al., 2015; Jorge et al., 2012; Mac Donald et al., 2011; Morey et al., 2013), similar to the civilian mTBI literature (but see Bazarian et al., 2013; Levin et al., 2010; Watts et al., 2014). However, findings of greater neurologic deficits in individuals exposed to combat-related mTBI than civilian mTBI (Ruff et al., 2012) provide some evidence for increased noxious effects due to blast and suggest the need for further studies comparing blast-induced mTBI and civilian TBI. Additionally, the high co-occurrence of PTSD in this population and the presence of white matter abnormalities associated with PTSD further complicate interpretation of structural brain connectivity (Davenport et al., 2015). Nonetheless, evidence from a recent post-mortem study of veterans suggests that blast exposure is associated with axonal pathology at the histological level (Ryu et al., 2014).

\section{Longitudinal Studies and Recovery after Injury}

Given the dynamic nature of traumatic axonal injury, DTI studies hold much promise for charting the progression and possible recovery of white matter injury over time. Interpretation of longitudinal findings is challenging, 
however, given that the role of primary and secondary injury processes will vary at different times post-injury and that there is as of yet no comprehensive model of TBI-associated diffusion changes over time. Changes in DTI metrics can be interpreted with reference to animal models of TBI (e.g., Mac Donald et al., 2007), but the time frame of pathophysiological responses in humans may not directly map onto that seen in animals. Additionally, given the known limitations in characterizing white matter in complex regions where fibers intersect, interpretation of longitudinal changes in such regions can be especially difficult. For instance, an FA increase over time that is consistent with axonal recovery or regeneration could also be observed when one of the crossing fiber populations degenerates, and the net result would be increased orientational coherence (e.g., Sidaros et al., 2008). Conversely, a lack of change in DTI metrics over time need not reflect absence of pathophysiological change. Evaluating white matter integrity in concussed football players 1-6 days postconcussion and again 6 months later, Henry et al. (2011) found similarly increased FA in the corpus callosum and corticospinal tract at both stages. Nonetheless, they speculated that distinct processes might be involved, with findings in the acute stage reflecting intracellular edema, and those in the chronic stage reflecting a loss of crossing fibers.

Notwithstanding these difficulties, several patterns of findings are emerging from recent longitudinal studies of TBI. Studies of mTBI that compare the acute or semi-acute stage to follow-up in the chronic stage have reported changes in DTI metrics consistent with continued evolution of the injury. For instance, evaluating veterans with blast-induced mTBI over a 6- to 12-month interval, Mac Donald et al. (2011) observed normalization of some DTI metrics (mean and radial diffusivity) but new abnormalities in others (axial diffusivity), suggesting that cellular edema and inflammation normalize, but axonal injury may persist. Other studies have provided evidence that DTI metrics reflecting axonal injury may partially normalize, suggesting a process of recovery that is incomplete (e.g., Arfanakis et al., 2002; Grossman et al., 2013; Mayer et al., 2010; but see Ling et al., 2012). Taken together, these studies leave open the possibility of continued axonal recovery over more extended time periods, although it is notable that persistent white matter changes have been observed even years after mTBI (e.g., Inglese et al., 2005).

There is also evidence for partial recovery of white matter injury in moderate/severe TBI (Kumar et al., 2010; Pal et al., 2012; Skoglund et al., 2008). For instance, examining a group of patients with frontal injury apparent on clinical neuroimaging, Pal and colleagues (2012) found significant increase in FA values in fronto-posterior and fronto-limbic tracts between 1 week and 6 months post-injury, although those values remained lower than in controls. Focusing specifically on the corpus callosum, Kumar et al. (2010) similarly reported an improvement in DTI metrics, albeit it not to the level of controls. Interestingly, the latter changes were apparent between the acute and 6-month follow-up scan, but there was no evidence for continued improvement at 2-year follow-up. To date, there is insufficient data to draw conclusions about the temporal window of recovery, but two group studies have indicated continued recovery up to a year (Sidaros et al., 2008) and 4 years after injury (Farbota et al., 2012). Perhaps most striking in this regard is a case study of a patient with severe TBI who emerged from a minimally conscious state 19 years post-injury and was subsequently scanned on two occasions 18 months apart (Voss et al., 2006). During that time, normalization of FA was seen in several regions, which was thought to represent axonal regrowth.

A striking counterpoint to the findings of partial recovery is evidence of continued deterioration of white matter in moderate/severe TBI. Greenberg et al. (2008) scanned patients with moderate to severe injury 4.5 months post-injury and then again on average 2 years later, and found increased deep frontal and temporal white matter impairment. Similarly, Wang et al. (2011) found, in a group of patients with mostly severe injury, that fiber tracts that showed structural abnormality acutely had further deteriorated on follow-up a year later. Moreover, additional regions that appeared normal initially also showed impaired DTI metrics. These findings suggest that white matter degeneration may continue for years beyond the initial injury (Bigler, 2013b). Such changes cannot be accounted for by the acute/sub-acute pathological effects of TBI, but likely reflect long-term degenerative mechanisms. Continued myelin degeneration (Meythaler et al., 2001), chronic neuroinflammation (Bigler, 2013a, 2013b; Johnson et al., 2013) and Wallerian degeneration as a result of axonal disconnection (Bendlin et al., 2008) have all been postulated as possible mechanisms. It has also been postulated that disuserelated negative plastic changes may further contribute to a downward negative spiral (Tomaszczyk et al., 2014).

Of interest, partial recovery and continued degeneration are not mutually exclusive. For instance, over a 10-month interval, Sidaros et al. (2008) reported an increase in DTI abnormalities in the posterior corpus callosum and cerebral peduncle, consistent with progressive atrophy, but a reduction of DTI abnormalities in the posterior limb of the internal capsule and centrum semiovale. They interpreted this finding as evidence that some axonal recovery or regrowth might be possible even against the backdrop of progressive atrophy. A similar conclusion was reached by Farbota et al. (2012; see also Bendlin et al., 2008). In their participants who were followed over 4 years, increases in FA were observed in the superior longitudinal fasciculus and optic radiation, concurrent with progressive FA loss in the corpus callosum. Why certain regions continue to deteriorate while others improve remains to be elucidated, but it has been speculated that both the extent of initial damage and tract-specific characteristics may play a role (Farbota et al., 2012).

\section{NEUROCOGNITIVE AND FUNCTIONAL CORRELATES OF WHITE MATTER CONNECTIVITY DISRUPTION}

\section{Neurocognitive Functions}

The cognitive functions typically affected by TBI such as executive functions, memory and attention depend on the 
connectivity of distributed, yet specific, neural networks. Thus, disruption of the associated white matter connections should have systematic consequences for cognition. Consistent with this notion, studies of TBI demonstrate that particular patterns of white matter abnormalities are associated with distinct profiles of cognitive impairment.

Some of the strongest evidence tying domain-specific cognitive dysfunction to discrete white matter pathways in TBI comes from examination of frontal, frontal-striatal, and frontal-parietal networks in association with executive functions. White matter tracts including the superior longitudinal fasciculus, corona radiata, internal capsule, and cingulum bundle connect frontal with posterior regions and allow for top-down control of activity in association cortices (Catani et al., 2013; Miller \& D'Esposito, 2005). Disruption of these white matter tracts has been associated with executive dysfunction in TBI. For instance, Kraus et al. (2007) found that reduced FA in the anterior corona radiata was associated with lower scores on a composite measure of executive functions. Kinnunen et al. (2011) found a relationship between mean diffusivity in the left superior frontal white matter and set shifting on the Trail Making Test. Furthermore, Little et al. (2010) found that thalamic-frontal disconnection was associated with attention and executive function deficits. Although studies examining the range of severity may be ideally suited to observe such correlations, studies limited to moderate/severe (Håberg et al., 2015; Palacios et al., 2012) or mild TBI (Lipton et al., 2009; Niogi et al., 2008) have reported analogous findings. For instance, in patients with acute mTBI, Lipton et al. (2009) found that FA abnormalities in frontal white matter, including the dorsolateral prefrontal cortex, were associated with omissions on the Continuous Performance Test and the number of failed trials on the Executive Maze Test. These studies support the notion that TBI-associated disconnection of frontal-posterior and frontal-subcortical networks has negative consequences for goal-directed behavior.

TBI-associated white matter abnormalities in specific tracts have also been linked to deficits in memory and learning. The fornix carries signals from the hippocampus to the basal forebrain and diencephalon and is critical for memory performance. Post-mortem pathology studies show that this region is commonly damaged in TBI (Blumbergs et al., 1995). As expected, disruption of the fornix is associated with poorer memory performance and learning (Kinnunen et al., 2011; Salmond et al., 2006). Similarly, disruption of other fiber tracts that carry information to and from the temporal lobes including the posterior cingulate and uncinate fasciculus have been associated with reduced memory performance in TBI or mTBI (Bendlin et al., 2008; Geary et al., 2010; Niogi et al., 2008; Salmond et al., 2006).

The corpus callosum is the largest collection of white matter fibers of the brain and, as noted above, it is also one of the most frequently damaged by TBI. Given the vast projections of the corpus callosum and its role in transmitting information across hemispheres, it is not surprising that lesions of the corpus callosum are associated with a diverse array of cognitive deficits. Diffusion abnormalities in this region have been associated with reduced performance in executive functions (Jorge et al., 2012; Kinnunen et al., 2011; Kraus et al., 2007), memory (Palacios et al., 2012; Salmond et al., 2006), and motor performance (Caeyenberghs et al., 2010; Farbota et al., 2012).

At a general level, the extant literature examining the cognitive consequences of white matter disruptions in TBI aligns well with expectations based on understanding of normal structure-function relations. However, it may be an oversimplification to assume that the structure-function relations observed in TBI simply represent a shift to the left on the curve of normal structure-function relations. For example, Niogi and colleagues (2008) demonstrated that both the control group and the TBI group showed a positive correlation between FA in the uncinate fasciculus and memory performance, and FA in the anterior corona radiata and attentional control. However, in other cases distinct relationships have been observed between DTI metrics and neurocognitive function in TBI and in the undamaged brain (Kinnunen et al., 2011; Little et al., 2010). Of interest, Kinnunen and colleagues (2011) found both patterns for different domains, with similar structure-function associations in TBI and healthy individuals with regard to memory, but distinct associations with regard to executive function. These findings place limits on the extent to which inferences from normal individuals can guide understanding of impairments following TBI.

\section{Postconcussion Syndrome}

Aside from neuropsychological performance, another focus of interest has been the link between white matter injury and subjective symptom reports, especially in individuals with mTBI. It is generally accepted that postconcussion symptoms (PCS) are associated with the acute neurologic effects of brain injury, and consistent with this view, studies have found an association between DTI metrics and PCS in the acute stage (e.g., Bazarian et al., 2007). The etiology of persistent PCS, however, is an issue of considerable debate given that many symptoms are non-specific and may also be associated with chronic pain and psychiatric conditions (McCrea et al., 2009). In light of this, it has been suggested that a variety of psychological, social, and motivational factors play a critical role in the maintenance of PCS, although some symptoms may be correlated with neuropathology (Bigler \& Maxwell, 2012). The availability of sensitive neuroimaging measures of axonal injury has provided an opportunity to directly examine the association between traumatic axonal injury and PCS in the subacute and chronic stage.

Consistent with the notion that PCS in the subacute or chronic stage are associated with neural abnormalities, several studies have shown a correlation between the magnitude of PCS and DTI metrics in various white matter tracts (Levin et al., 2010; Ling et al., 2012; Smits et al., 2011; Yeh et al., 2014; but see Petrie et al., 2014). As an example, 
Yeh and colleagues (2014) found that postconcussive symptom severity was associated with decreased FA in the cingulum bundle, corpus callosum, corona radiata, internal capsule, and frontal fibers. The diffuse white matter abnormalities observed in this study likely reflect the wideranging symptoms that comprise PCS. Other studies have used a group approach to compare individuals with and without PCS; such studies have yielded mixed results, with some studies showing group differences (Bouix et al., 2013; Hartikainen et al., 2010; Messé et al., 2012) and others not (Lange et al., 2012, 2015; Wäljas et al., 2014). Reasons for these inconsistencies are unclear, although studies comparing dichotomous groups may inherently have lower power to detect white matter associations than do correlational studies.

The observation of structural white matter changes associated with PCS reports beyond the acute stage can be interpreted in several ways. On the one hand, it is consistent with the notion that persistent PCS reflect direct neurological effects of mTBI. On the other hand, equally plausible is the possibility that TBI-associated white matter abnormalities play a role in the development or maintenance of mental health conditions including PTSD (Bazarian et al., 2013; Costanzo et al., 2014) and depression (Matthews et al., 2011), which in turn may be associated with chronic PCS. To date, there are no longitudinal studies examining the link between white matter, PCS, and the development of psychopathology. Such studies may be helpful in further probing the relationship between axonal injury, psychiatric symptoms, and PCS at different time frames following TBI. In this context, consideration of separate clusters of PCS will be important, as such clusters may have a distinct origin and longitudinal course.

\section{WHITE MATTER INJURY AS A MARKER OF TBI OUTCOME}

Partial normalization of DTI metrics has been associated with favorable clinical outcome (Sidaros et al., 2008), reduced somatic complaints (Ling et al., 2012), recovery from hemiparesis (Skoglund et al., 2008), and improved neuropsychological functioning (Bendlin et al., 2008; Farbota et al., 2012; Kumar et al., 2010; Newcombe et al., 2015). Conversely, deterioration of white matter microstructure in the first 6 months after injury has been associated with worse clinical outcome (Ljungqvist et al., 2011).

Given the evidence that white matter changes are correlated with clinical and cognitive recovery, it would appear that quantification of white matter injury holds considerable promise as a marker of TBI outcome. Indeed, evidence suggests that not only visible white matter abnormalities (Matsukawa et al., 2011) but also more subtle traumatic axonal injury, as indexed by diffusion measures in the acute or subacute phase, is associated with unfavorable outcome (Hou et al., 2007; Messé et al., 2011; Perlbarg et al., 2009; Sidaros et al., 2008; Yuh et al., 2014). In one study, increased apparent diffusion in the corpus callosum was associated with unfavorable outcome as measured by the Glasgow Outcome scale (Hou et al., 2007). In two other studies, reduced FA in the cerebral peduncle (Perlbarg et al., 2009; Sidaros et al., 2008) and the posterior corpus callosum, posterior limb of the internal capsule, and inferior longitudinal fasciculus (Perlbarg et al., 2009) were predictive of poor outcome. In both cases, DTI improved the prediction accuracy in comparison to that based on clinical data. Findings regarding the prognostic utility of DTI metrics for cognitive outcome are limited, although there is some suggestive evidence that various DTI metrics are associated with subsequent memory (Wang et al., 2011), complex executive function (Miles et al., 2008), and speeded sensory motor function (Moen et al., 2014).

In light of the heterogeneity of patients in these studies, the identification of consistent patterns of injury associated with outcome is encouraging. However, more work is clearly needed to evaluate whether particular DTI metrics have superior prognostic value, and importantly, it remains to be determined whether such evidence can be used for classification at the individual level (e.g., Hellyer et al., 2013; Perlbarg et al., 2009). As described in the next section, it is likely that incorporation of information about functional connectivity in addition to information about structural connectivity will be helpful in this endeavor.

\section{TBI-ASSOCIATED FUNCTIONAL CONNECTIVITY ALTERATIONS}

The fact that diffuse traumatic injury commonly disrupts white matter tracts that connect distant brain regions naturally raises the question as to how TBI impacts the functioning of large-scale brain networks that are critical for cognition and behavior. While investigation of intrinsic connectivity networks in TBI is still a relatively novel endeavor (for review see Sharp et al., 2014), alterations have been shown in several resting state networks including the default mode network (DMN; Bonelle et al., 2011; Messé et al., 2013; Sharp et al., 2011; Stevens et al., 2012; Zhou et al., 2012), the thalamic network (Tang et al., 2011; Zhou et al., 2014), motor network (Shumskaya et al., 2012), salience network (Bonelle et al., 2012; Ham et al., 2014; Jilka et al., 2014), and executive control network (Mayer et al., 2011; Shumskaya et al., 2012), as well as interhemispheric functional connectivity (Marquez de la Plata et al., 2011).

\section{Default Mode Network}

A strong focus of functional connectivity studies in TBI has been on the DMN. This network is critically involved in internal thought, when external cognitive demands are minimized as during "resting" state scanning, but needs to be inhibited during tasks that require attention to the external environment. Thus, its integrity is critical to cognitive task performance. Because the DMN involves long-coursing parietofrontal and interhemispheric projecting axons, it is vulnerable to TBI. Several studies have demonstrated altered functional connectivity within the DMN, both in moderate/severe and in mild TBI, and such alterations have been linked to the presence of postconcussion symptoms (Messé et al., 2013; Stevens et al., 2012; 
Zhou et al., 2012) and cognitive changes (Bonelle et al., 2011; Sharp et al., 2011). In patients with moderate to severe TBI, increased functional connectivity between core nodes of the DMN is commonly observed, and especially with the posterior cingulate and precuneus regions (Hillary et al., 2014; Palacios et al., 2013; Sharp et al., 2011; but see Arenivas et al., 2014; Pandit et al., 2013). However, there is also evidence that TBI is associated with decreased connectivity in some nodes of the DMN and increased connectivity in other nodes (Venkatesan et al., 2015), possibly reflecting the distinct role of different nodes within the network. Similar non-uniformity of network changes has been observed in mild TBI (Johnson et al., 2012; Stevens et al., 2012; Zhou et al., 2012), while other studies in mild TBI have observed generally decreased connectivity (Costanzo et al., 2014; Mayer et al., 2011). Alterations in the DMN have been linked to structural damage to the cingulum bundle (Costanzo et al., 2014; Palacios et al., 2013) and corpus callosum (Sharp et al., 2011).

Aside from alterations in the DMN itself, there is considerable evidence for impaired coordination between the DMN and task-positive networks such as the executive control network (Sours et al., 2013; Venkatesan et al., 2015) and salience network (Sours et al., 2013). The salience network, in particular, has been shown to play an important role in shifting between internally and externally directed states, and as such, may be critical for balancing network function necessary for efficient cognition. Focusing specifically on interactions between the salience network and DMN during tasks requiring motor control, Sharp and colleagues (Bonelle et al., 2012; Jilka et al., 2014) found that patients with TBI-associated cognitive control problems failed to suppress DMN activity during task performance (Bonelle et al., 2012), and that this failure was associated with an inability to transiently upregulate functional connectivity between these networks (Jilka et al., 2014). Furthermore, the breakdown of this network interaction was related to the extent of structural damage within the salience network, specifically in a white matter tract connecting the right anterior insula with the pre-SMA/dorsal anterior cingulate.

\section{Graph Theory}

Recently, whole-brain connectivity patterns have also been analyzed using graph theoretical analyses that characterize networks graphically as a set of interacting nodes (groups of neurons) connected by edges (white matter tracts), and thus more closely resemble the distributed network architecture of the brain. Brain networks are characterized by small-world topology, an architecture in which densely connected local modules are combined with sparse long distance connections that allow for communication across modules. Several studies have demonstrated a reduction of small-world properties in TBI, including decreased network efficiency (Nakamura et al., 2009; Pandit et al., 2013), modularity (Messé et al., 2013), overall connectivity (Pandit et al., 2013) and betweennetwork connectivity (Han et al., 2014). These alterations are consistent with disruption of long coursing fibers as a result of traumatic axonal injury. However, as noted above, increased local connectivity early in the course of recovery, especially in core networks known as the "rich club", has also been documented (Hillary et al., 2014). Evidence is also emerging suggesting that graph metrics relate to behavioral impairment (Caeyenberghs et al., 2012) and may be predictive of response to cognitive training (Arnemann et al., 2015). However, which metrics will be most informative in characterizing alterations in brain structure and function remains to be determined.

\section{Network Analysis of Injury Recovery}

Given the dynamic changes during recovery following TBI, the time post-injury when network analysis is performed is critical to understanding the neuropsychological significance of findings. To date, few studies have charted longitudinal changes in network function. Hillary et al. (2011) demonstrated in moderate/severe TBI that during a critical period of recovery, between 3 and 6 months post-injury, resting-state connectivity increased in the DMN and decreased in the fronto-parietal control network (Hillary et al., 2011). Moreover, connectivity between each of these networks and the salience network increased, potentially allowing for more efficient transition between networks. In a more recent study from the same group (Venkatesan et al., 2015), patients with moderate/severe TBI assessed during the subacute period were scanned again on average 2 years post-injury. The authors observed reduced DMN connectivity from the posterior cingulate and increased anti-correlations with taskpositive regions, consistent with normalization of network connectivity (see also Nakamura et al., 2009). However, at the same time, DMN hyperconnectivity with hippocampal nodes became more pronounced. The significance of this finding is unclear, although the authors suggested a potential compensatory mechanism. However, these findings clearly illustrate the complexity of network changes and challenge the notion of uniform recovery.

Another factor that may contribute to the complex pattern of network dysfunction is the heterogeneous pathology in TBI and the extent to which focal injury may interact with diffuse white matter injury. In this regard, damage to critical hubs that link different networks may have far-reaching consequences that go well beyond the function of any particular network, and may be an important mechanism underlying long term cognitive impairment (Fagerholm et al., 2015).

\section{OUTSTANDING ISSUES}

Although there are emerging patterns that are fairly consistent across TBI studies, some areas remain in need of further exploration. One such area concerns the nature of the white abnormalities in the acute and subacute phase following mTBI. Of note, several studies have shown abnormalities in DTI metrics in the opposite direction of what is typically seen, namely reporting increased FA or decreased MD/ADC (Bazarian et al., 2007; Mayer et al., 2010; Wäljas et al., 2014). The mechanism 
underlying these unexpected findings is unclear, although it has been suggested they may reflect the presence of neuroinflammation. Consistent with this notion, Marmorou and colleagues (2006) found that ADC was decreased relative to healthy subjects only in individuals with TBI-induced axonal injury who had brain swelling and edema, but not in a group with axonal injury without brain swelling. Others have suggested that such departures from the typical pattern of injury may reflect plasticity or other compensatory mechanisms (Lipton et al., 2012). Animal models have demonstrated that increased FA does occur following TBI, especially within the more mild range of injury (Begonia et al., 2014).

Another consideration relates to the spatial distribution of white matter abnormalities following mTBI. As noted above, some regions are particularly vulnerable to injury. Nonetheless, some studies have failed to find abnormalities in specific white matter tracts, particularly in the blast-related mTBI literature (Bazarian et al., 2013; Ilvesmaki et al., 2014; Levin et al., 2010; Watts et al., 2014). These diverse outcomes likely reflect heterogeneity in the distribution of mTBI pathology across individuals, associated with variable direction and location of injury forces. For instance, using methods optimized for assessment of individual mTBI patients, Lipton et al. (2012) showed that the pattern of white matter abnormalities was unique for each individual. Several methods for examining spatial heterogeneity of injury have recently been adopted in both cross-sectional (see Davenport et al., 2012; Hayes et al., 2015; Jorge et al., 2012; Lipton et al., 2012; Taber et al., 2015) and longitudinal studies (Ling et al., 2012) with the common feature that these approaches do not limit damage to a particular spatial boundary. These methods have revealed that white matter damage is detected when spatial heterogeneity is taken into account rather than expecting that damage to white matter tracts will overlap in all individuals with mTBI.

Relatedly, not all studies have found relationships between specific white matter tracts and cognitive function (e.g., Miles et al., 2008). Such negative findings may also be due to a failure to capture the spatial heterogeneity that characterizes axonal injury associated with TBI. As an alternative, several studies have used summary measures representing the total number of abnormal white matter regions in relationship with cognitive function. Niogi et al. (2008) found that the number of abnormal white matter regions was positively associated with slowed reaction time. Krause et al. (2007) also examined total number of abnormal ROIs, which was associated with reduced executive function. Bazarian et al. (2007) calculated a percentile index, showing that FA values in the first percentile were lower in the TBI group and were associated with visuomotor speed and impulse control. Such an approach may also be helpful in assessing white matter correlates of cognitive processes that are likely to be more diffusely mediated, such as processing speed (but see Kinnunen et al., 2011; Farbota et al., 2012 for specific white matter tract associations).

With regard to rs-fcMRI results, it remains poorly understood how disruption of white matter tracts may give rise to increased connectivity among remaining nodes. On the one hand, it has been suggested that hyperconnectivity between nodes is a generalized response to neural disruption (Hillary et al., 2015). Consistent with this notion are findings that hyperconnectivity is associated with negative outcomes such as cognitive fatigue (Mayer et al., 2011) and postconcussive symptoms (Shumskaya et al., 2012). On the other hand, hyperconnectivity may reflect compensatory connectivity within pathways that are still connected to maintain function (Palacios et al., 2013; Sharp et al., 2011). For instance, because the DMN is typically anti-correlated with task-positive networks, it has been suggested that upregulation of DMN connectivity might tune the network so as to allow rapid deactivation required for task performance (Sharp et al., 2011). Consistent with this view are findings of a positive correlation between DMN connectivity and cognitive outcome (Bonelle et al., 2011; Sharp et al., 2011). Additional research is necessary to understand the relationship between increased functional connectivity and structural integrity loss in different networks.

\section{CONCLUSIONS AND FUTURE DIRECTIONS}

Since the time of Geschwind's seminal work, great progress has been made in understanding connectivity among brain regions that work in concert to bring about goal related behavior, as well as the consequences of damage to these networks as a result of traumatic axonal injury. Although post-mortem and histology work has been pivotal in understanding the TBI-associated abnormalities to network connections, recent advances in neuroimaging have helped to rapidly move the field forward. As TBI is primarily a white matter disorder, the advent of diffusion imaging has allowed visualization and quantification of disrupted white matter connections and axonal injury. Our review of the literature suggests that TBI, even of mild severity, is associated with acute and chronic white matter abnormalities that likely involve the loss of axonal integrity and myelin, and non-specific mechanisms of injury including inflammation and edema. Although there may be some tracts that are particularly vulnerable to axonal injury such as the corpus callosum, TBI is a spatially heterogeneous injury, characterized by diffuse damage to white matter fibers. Longitudinal studies have demonstrated that, although there is evidence for axonal recovery, persistent white matter abnormalities are observed in the chronic state of injury, particularly in more severe injuries. These white matter abnormalities are not without consequences; the review of the literature reveals that structural abnormalities are associated most often with executive dysfunction and reduced memory performance.

Although greater work is necessary to examine the link between TBI and mental health, there is strong preliminary evidence that TBI and psychiatric conditions play an important and possibly synergistic role in cognitive and functional outcomes.

The current review highlights the fact that TBI is a complex and heterogeneous injury. As mentioned above, 
neuroimaging methods that capture individual injury variability are more likely to uncover white matter abnormalities than measures that examine particular tracts, and may be more useful in determining the functional significance of the injury. Analogously, in the context of blast-related mTBI, recent evidence suggest that the neuropsychological sequelae in the chronic stage may be more apparent by evaluating at the individual subject level the number of tests with abnormal performance than by evaluating group differences in specific tests (Mac Donald et al., 2015). Whether such a cognitive load measure is associated with measures of heterogeneous white matter abnormality remains to be determined. Conversely, examination of patient groups who are more homogeneous in terms of behavioral symptoms or neuropsychological profiles may improve detection of damage to specific brain regions (e.g., Sorg et al., 2013).

Although the emphasis on examining white matter networks in TBI is natural considering that the primary pathology is axonal injury, examination of intrinsic connectivity networks has allowed for a more comprehensive understanding of the effects of TBI on brain function. Investigations of core intrinsic connectivity networks in TBI have shown alterations in functional connectivity and reduction in small-worldness of large-scale networks, and disturbed coordination between the DMN and task-positive networks. However, as illustrated by functional connectivity changes during task performance, network function is not constant over time. How natural fluctuations in network connectivity may be altered by TBI represents a promising area for future study.

Another area ripe for future work concerns the integration of functional and structural MRI data. With the advent of novel multivariate statistical approaches, it is now possible to directly examine the effect of structural disconnection on large-scale neural dynamics (e.g., Hellyer et al., 2015). Additionally, relationships between white matter integrity and functional connectivity at the network level are beginning to be explored (e.g., Stevens et al., 2012). Alterations in these relationships as a result of TBI may provide valuable information about the global effects of injury that cannot be obtained by each method separately.

An important challenge for future work is to determine how information about structural and functional connectivity changes can be used clinically for diagnosis and outcome prediction in individual patients. Irimia and colleagues (2012, 2014) have taken a step in this direction by using multimodal imaging to create individual connectivity matrices which specify fiber density between every pair of regions in the brain. Such matrices can be used to chart longitudinal changes and in combination with neuropsychological and behavioral measures, may provide important insights into recovery and response to treatment. Ultimately, such information may help to direct clinical interventions for rehabilitation.

The use of machine-learning approaches provides another promising avenue for the personalized application of neuroimaging methods. Using a classifier trained on data that are already diagnostically labeled, diagnostic classifications can then be made about new data and predictions generated about cognitive or behavioral sequelae of the injury. These methods are in their infancy, but have already been successfully applied to DTI data (Hellyer et al., 2013) as well as graph metrics (Fagerholm et al., 2015). Future work that examines how TBI affects the dynamic integration of structural and functional networks holds promise for elucidating how the brain is altered, repairs itself, and degenerates following TBI.

\section{ACKNOWLEDGMENTS}

There are no financial disclosures or conflicts of interest to report for any of the authors. This work was supported in part by the Department of Veterans Affairs, Veterans Health Administration, Rehabilitation Research and Development Service Award number I21 RX001594, the National Center for PTSD and the VA Clinical Science Research and Development Service.

\section{REFERENCES}

Adams, J.H., Doyle, D., Ford, I., Gennarelli, T.A., Graham, D.I., \& McLellan, D.R. (1989). Diffuse axonal injury in head injury: Definition, diagnosis and grading. Histopathology, 15, 49-59.

Aoki, Y., Inokuchi, R., Gunshin, M., Yahagi, N., \& Suwa, H. (2012). Diffusion tensor imaging studies of mild traumatic brain injury: A meta-analysis. Journal of Neurology, Neurosurgery, \& Psychiatry, 83, 870-876.

Arenivas, A., Diaz-Arrastia, R., Spence, J., Cullum, C., Krishnan, K., Bosworth, C., ... Marquez de la Plata, C. (2014). Three approaches to investigating functional compromise to the default mode network after traumatic axonal injury. Brain Imaging and Behavior, 8, 407-419.

Arfanakis, K., Haughton, V.M., Carew, J.D., Rogers, B.P., Dempsey, R.J., \& Meyerand, M.E. (2002). Diffusion tensor MR imaging in diffuse axonal injury. AJNR: American Journal of Neuroradiology, 23, 794-802.

Arnemann, K.L., Chen, A.J.W., Novakovic-Agopian, T., Gratton, C., Nomura, E.M., \& D'Esposito, M. (2015). Functional brain network modularity predicts response to cognitive training after brain injury. Neurology, 84, 1568-1574.

Ashwal, S., Tong, K.A., Ghosh, N., Bartnik-Olson, B., \& Holshouser, B.A. (2014). Application of advanced neuroimaging modalities in pediatric traumatic brain injury. Journal of Child Neurology, 29, 1704-1717.

Bazarian, J.J., Donnelly, K., Peterson, D.R., Warner, G.C., Zhu, T., \& Zhong, J. (2013). The relation between posttraumatic stress disorder and mild traumatic brain injury acquired during Operations Enduring Freedom and Iraqi Freedom. Journal of Head Trauma Rehabilitation, 28, 1-12.

Bazarian, J.J., Zhong, J., Blyth, B., Zhu, T., Kavcic, V., \& Peterson, D. (2007). Diffusion tensor imaging detects clinically important axonal damage after mild traumatic brain injury: A pilot study. Journal of Neurotrauma, 24, 1447-1459.

Bazarian, J.J., Zhu, T., Blyth, B., Borrino, A., \& Zhong, J. (2012). Subject-specific changes in brain white matter on diffusion tensor imaging after sports-related concussion. Magnetic Resonance Imaging, 30, 171-180. 
Begonia, M., Prabhu, R., Liao, J., Whittington, W., Claude, A., Willeford, B., Wardlaw, J., ... Williams, L. (2014). Quantitative analysis of brain microstructure following mild blunt and blast trauma. Journal of Biomechanics, 47(15), 3704-3711.

Bendlin, B.B., Ries, M.L., Lazar, M., Alexander, A.L., Dempsey, R.J., Rowley, H.A., ... Johnson, S.C. (2008). Longitudinal changes in patients with traumatic brain injury assessed with diffusion-tensor and volumetric imaging. Neuroimage, 42, 503-514.

Bigler, E.D. (2001). The lesion (s) in traumatic brain injury: Implications for clinical neuropsychology. Archives of Clinical Neuropsychology, 16, 95-131.

Bigler, E.D. (2013a). Neuroinflammation and the dynamic lesion in traumatic brain injury. Brain, 136, 9-11.

Bigler, E.D. (2013b). Traumatic brain injury, neuroimaging, and neurodegeneration. Frontiers in Human Neuroscience, 7, 395.

Bigler, E.D., Abildskov, T.J., Wilde, E.A., McCauley, S.R., Li, X., Merkley, T.L., ... Levin, H.S. (2010). Diffuse damage in pediatric traumatic brain injury: A comparison of automated versus operatorcontrolled quantification methods. Neuroimage, 50, 1017-1026.

Bigler, E.D., \& Maxwell, W.L. (2012). Neuropathology of mild traumatic brain injury: Relationship to neuroimaging findings. Brain Imaging and Behavior, 6, 108-136.

Blumbergs, P.C., Scott, G., Vis, J.M., Wainwright, H., Simpson, D.A., \& McLean, A.J. (1995). Topography of axonal injury as defined by amyloid precursor protein and the sector scoring method in mild and severe closed head injury. Journal of Neurotrauma, 12(4), 565-572.

Bonelle, V., Ham, T., Leech, R., Kinnunen, K., Mehta, M., Greenwood, R., \& Sharp, D. (2012). Salience network integrity predicts default mode network function after traumatic brain injury. Proceedings of the National Academy of Sciences of the United States of America, 109, 4690-4695.

Bonelle, V., Leech, R., MKinnunen, K., Ham, T., Beckmann, C., De Boissezon, X., ... Sharp, D. (2011). Default mode network connectivity predicts sustained attention deficits after traumatic brain injury. The Journal of Neuroscience, 31, 13442-13451.

Bouix, S., Pasternak, O., Rathi, Y., Pelavin, P.E., Zafonte, R., \& Shenton, M.E. (2013). Increased gray matter diffusion anisotropy in patients with persistent post-concussive symptoms following mild traumatic brain injury. PLoS One, 8(6), e66205.

Bramlett, H.M., \& Dietrich, W.D. (in press). Long-term consequences of traumatic brain injury: Current status of potential mechanisms of injury and neurological outcomes. Journal of Neurotrauma. doi: 10.1089/neu.2014.3352.

Caeyenberghs, K., Leemans, A., Geurts, M., Taymans, T., Vander Linden, C., Smits-Engelsman, B.C.M., ... Swinnen, S.P. (2010). Brain-behavior relationships in young traumatic brain injury patients: Fractional anisotropy measures are highly correlated with dynamic visuomotor tracking performance. Neuropsychologia, 48 (5), 1472-1482.

Caeyenberghs, K., Leemans, A., Heitger, M.H., Leunissen, I., Dhollander, T., Sunaert, S., ... Swinnen, S.P. (2012). Graph analysis of functional brain networks for cognitive control of action in traumatic brain injury. Brain, 135(4), 1293-1307.

Catani, M. (2005). The rises and falls of disconnection syndromes. Brain, 128(10), 2224-2239.

Catani, M., Dell'Acqua, F., \& Thiebaut de Schotten, M. (2013). A revised limbic system model for memory, emotion and behaviour. Neuroscience \& Biobehavioral Reviews, 37, 1724-1737.

Catani, M., \& Thiebaut de Schotten, M. (2008). A diffusion tensor imaging tractography atlas for virtual in vivo dissections. Cortex, $44,1105-1132$.
Choudhri, A.F., Chin, E.M., Blitz, A.M., \& Gandhi, D. (2014) Diffusion tensor imaging of cerebral white matter. Radiologic Clinics of North America, 52, 413-425.

Cole, D.M., Smith, S.M., \& Beckmann, C.F. (2010). Advances and pitfalls in the analysis and interpretation of resting-state FMRI data. Frontiers in Systems Neuroscience, 4, 8.

Corbetta, M. (2012). Functional connectivity and neurological recovery. Developmental Psychobiology, 54, 239-253.

Costanzo, M.E., Chou, Y.-Y., Leaman, S., Pham, D.L., Keyser, D., Nathan, D.E., ... Roy, M.J. (2014). Connecting combat-related mild traumatic brain injury with posttraumatic stress disorder symptoms through brain imaging. Neuroscience Letters, 577, 11-15.

Davenport, N.D., Lim, K.O., Armstrong, M.T., \& Sponheim, S.R. (2012). Diffuse and spatially variable white matter disruptions are associated with blast-related mild traumatic brain injury. Neuroimage, 59, 2017-2024.

Davenport, N.D., Lim, K.O., \& Sponheim, S.R. (2015). White matter abnormalities associated with military PTSD in the context of blast TBI. Human Brain Mapping, 36(3), 1053-1064.

Fagerholm, E.D., Hellyer, P.J., Scott, G., Leech, R., \& Sharp, D.J. (2015). Disconnection of network hubs and cognitive impairment after traumatic brain injury. Brain, 138(6), 1696-1709.

Fakhran, S., Yaeger, K., \& Alhilali, L. (2013). Symptomatic white matter changes in mild traumatic brain injury resemble pathologic features of early Alzheimer dementia. Radiology, 269, 249-257.

Farbota, K.D., Bendlin, B.B., Alexander, A.L., Rowley, H.A., Dempsey, R.J., \& Johnson, S.C. (2012). Longitudinal diffusion tensor imaging and neuropsychological correlates in traumatic brain injury patients. Frontiers in Human Neuroscience, 6, 160.

Gale, S.D., Johnson, S.C., Bigler, E.D., \& Blatter, D.D. (1995). Nonspecific white matter degeneration following traumatic brain injury. Journal of the International Neuropsychological Society, 1(1), 17-28.

Gardner, A., Kay-Lambkin, F., Stanwell, P., Donnelly, J., Williams, W.H., Hiles, A., ... Jones, D.K. (2012). A systematic review of diffusion tensor imaging findings in sports-related concussion. Journal of Neurotrauma, 29, 2521-2538.

Geary, E.K., Kraus, M.F., Pliskin, N.H., \& Little, D.M. (2010). Verbal learning differences in chronic mild traumatic brain injury. Journal of the International Neuropsychological Society, 16, 506.

Gentry, L.R., Thompson, B., \& Godersky, J.C. (1988). Trauma to the corpus callosum: MR features. AJNR: American Journal of Neuroradiology, 9(6), 1129-1138.

Geschwind, N. (1965). Disconnexion syndromes in animals and man. Brain, 88(3), 585-585.

Goetz, P., Blamire, A., Rajagopalan, B., Cadoux-Hudson, T., Young, D., \& Styles, P. (2004). Increase in apparent diffusion coefficient in normal appearing white matter following human traumatic brain injury correlates with injury severity. Journal of Neurotrauma, 21, 645-654.

Greenberg, G., Mikulis, D.J., Ng, K., DeSouza, D., \& Green, R.E. (2008). Use of diffusion tensor imaging to examine subacute white matter injury progression in moderate to severe traumatic brain injury. Archives of Physical Medicine and Rehabilitation, 89, S45-S50.

Grossman, E.J., Jensen, J.H., Babb, J.S., Chen, Q., Tabesh, A., Fieremans, E., ... Grossman, R.I. (2013). Cognitive impairment in mild traumatic brain injury: A longitudinal diffusional kurtosis and perfusion imaging study. AJNR: American Journal of Neuroradiology, 34, 951-957. 
Håberg, A., Olsen, A., Moen, K., Schirmer-Mikalsen, K., Visser, E., Finnanger, T., ... Eikenes, L. (2015). White matter microstructure in chronic moderate-to-severe traumatic brain injury: Impact of acute-phase injury-related variables and associations with outcome measures. Journal of Neuroscience Research, 93, $1109-1126$.

Ham, T.E., Bonnelle, V., Hellyer, P., Jilka, S., Robertson, I.H., Leech, R., \& Sharp, D.J. (2014). The neural basis of impaired self-awareness after traumatic brain injury. Brain, 137(2), 586-597.

Han, K., Mac Donald, C., Johnson, A., Barnes, Y., Wierzechowski, L., Zonies, D., ... Brody, D. (2014). Disrupted modular organization of resting-state cortical functional connectivity in U.S. military personnel following concussive 'mild' blast-related traumatic brain injury. Neuroimage, 84, 76-96.

Hartikainen, K.M., Wäljas, M., Isoviita, T., Dastidar, P., Liimatainen, S., Solbakk, A.-K., ... Öhman, J. (2010). Persistent symptoms in mild to moderate traumatic brain injury associated with executive dysfunction. Journal of Clinical and Experimental Neuropsychology, 32, 767-774.

Hayes, J.P., Miller, D.R., Lafleche, G., Salat, D.H., \& Verfaellie, M. (2015). The nature of white matter abnormalities in blast-related mild traumatic brain injury. Neuroimage: Clinical, 8, 148-156.

Hayes, J.P., Morey, R.A., \& Tupler, L.A. (2012). A case of frontal neuropsychological and neuroimaging signs following multiple primary-blast exposure. Neurocase, 18, 258-269.

Hellyer, P.J., Leech, R., Ham, T.E., Bonnelle, V., \& Sharp, D.J. (2013). Individual prediction of white matter injury following traumatic brain injury: Multivariate analysis of white matter after TBI. Annals of Neurology, 73, 489-499.

Hellyer, P.J., Scott, G., Shanahan, M., Sharp, D.J., \& Leech, R. (2015). Cognitive flexibility through metastable neural dynamics is disrupted by damage to the structural connectome. The Journal of Neuroscience, 35(24), 9050-9063.

Henry, L.C., Tremblay, J., Tremblay, S., Lee, A., Brun, C., Lepore, N., ... Lassonde, M. (2011). Acute and chronic changes in diffusivity measures after sports concussion. Journal of Neurotrauma, 28, 2049-2059.

Hillary, F., Rajtmajer, S., Roman, C., Medaglia, J., SlocombDluzen, J., Calhoun, V., ... Wylie, G. (2014). The rich get richer: Brain injury elicits hyperconnectivity in core subnetworks. PLoS One, 9, 1-15.

Hillary, F., Roman, C., Venkatesan, U., \& Rajtmajer, S. (2015). Hyperconnectivity is a fundamental response to neurological disruption. Neuropsychology, 29, 59-75.

Hillary, F., Slocomb, J., Hills, E., Fitzpatrick, N., Medaglia, J., Wang, J., ... Wylie, G. (2011). Changes in resting connectivity during recovery from severe traumatic brain injury. International Journal of Psychophysiology, 82, 115-123.

Hou, D.J., Tong, K.A., Ashwal, S., Oyoyo, U., Joo, E., Shutter, L. \& Obenaus, A. (2007). Diffusion-weighted magnetic resonance imaging improves outcome prediction in adult traumatic brain injury. Journal of Neurotrauma, 24, 1558-1569.

Huisman, T.A., Schwamm, L.H., Schaefer, P.W., Koroshetz, W.J., Shetty-Alva, N., Ozsunar, Y., ... Sorensen, A.G. (2004). Diffusion tensor imaging as potential biomarker of white matter injury in diffuse axonal injury. AJNR: American Journal of Neuroradiology, 25(3), 370-376.

Hulkower, M.B., Poliak, D.B., Rosenbaum, S.B., Zimmerman, M.E., \& Lipton, M.L. (2013). A decade of DTI in traumatic brain injury: 10 years and 100 articles later. AJNR: American Journal of Neuroradiology, 34, 2064-2074.
Ilvesmaki, T., Luoto, T.M., Hakulinen, U., Brander, A., Ryymin, P., Eskola, H., ... Ohman, J. (2014). Acute mild traumatic brain injury is not associated with white matter change on diffusion tensor imaging. Brain, 137, 1876-1882.

Inglese, M., Makani, S., Johnson, G., Cohen, B.A., Silver, J.A., Gonen, O., \& Grossman, R.I. (2005). Diffuse axonal injury in mild traumatic brain injury: A diffusion tensor imaging study. Journal of Neurosurgery, 103, 298-303.

Irimia, A., Chambers, M.C., Torgerson, C.M., Filippou, M., Hovda, D.A., Alger, J.R., ... Kikinis, R. (2012). Patienttailored connectomics visualization for the assessment of white matter atrophy in traumatic brain injury. Frontiers in Neurology, 3,10 .

Irimia, A., Goh, S.-Y., Torgerson, C., Vespa, P., \& Van Horn, J. (2014). Structural and connectomic neuroimaging for the personalized study of longitudinal alterations in cortical shape, thickness and connectivity after traumatic brain injury. Journal of Neurosurgical Sciences, 58(3), 129.

Jilka, S., Scott, G., Ham, T., Pickering, A., Bonelle, V., Braga, R., ... Sharp, D. (2014). Damage to the salience network and interactions with the default mode network. The Journal of Neuroscience, 34, 10798-10807.

Johnson, B., Zhang, K., Gay, M., Horovitz, S., Hallett, M., Sebastianelli, W., \& Slobounov, S. (2012). Alteration of brain default network in subacute phase of injury in concussed individuals: Resting-state fMRI study. Neuroimage, 59, 511-518.

Johnson, V.E., Stewart, J.E., Begbie, F.D., Trojanowski, J.Q., Smith, D.H., \& Stewart, W. (2013). Inflammation and white matter degeneration persist for years after a single traumatic brain injury. Brain, 136, 28-42.

Jones, D. (2008). Studying connections in the living human brain with diffusion MRI. Cortex, 44, 936-952.

Jones, D.K., \& Cercignani, M. (2010). Twenty-five pitfalls in the analysis of diffusion MRI data. NMR in Biomedicine, 23(7), 803-820.

Jorge, R.E., Acion, L., White, T., Tordesillas-Gutierrez, D., Pierson, R., Crespo-Facorro, B., \& Magnotta, V.A. (2012). White matter abnormalities in veterans with mild traumatic brain injury. American Journal of Psychiatry, 169, 1284-1291.

Kennedy, M.R.T., Wozniak, J.R., Muetzel, R.L., Mueller, B.A., Chiou, H.-H., Pantekoek, K., \& Lim, K.O. (2009). White matter and neurocognitive changes in adults with chronic traumatic brain injury. Journal of the International Neuropsychological Society, 15, 130-136.

Kim, N., Branch, C.A., Kim, M., \& Lipton, M.L. (2013). Whole brain approaches for identification of microstructural abnormalities in individual patients: Comparison of techniques applied to mild traumatic brain injury. PLoS One, 8, e59382.

Kinnunen, K.M., Greenwood, R., Powell, J.H., Leech, R., Hawkins, P.C., Bonnelle, V., ... Sharp, D.J. (2011). White matter damage and cognitive impairment after traumatic brain injury. Brain, 134, 449-463.

Koerte, I.K., Ertl-Wagner, B., Reiser, M., Zafonte, R., \& Shenton, M.E. (2012). White matter integrity in the brains of professional soccer players without a symptomatic concussion. Journal of the American Medical Association, 308(18), 1859-1861.

Koerte, I.K., Kaufmann, D., Hartl, E., Bouix, S., Pasternak, O., Kubicki, M., ... Shenton, M.E. (2012). A prospective study of physician-observed concussion during a varsity university hockey season: White matter integrity in ice hockey players. Part 3 of 4. Neurosurgical Focus, 33, E3. 
Kraus, M.F., Susmaras, T., Caughlin, B.P., Walker, C.J., Sweeney, J.A., \& Little, D.M. (2007). White matter integrity and cognition in chronic traumatic brain injury: A diffusion tensor imaging study. Brain, 130, 2508-2519.

Kumar, R., Saksena, S., Husain, M., Srivastava, A., Rathore, R.K., Agarwal, S., \& Gupta, R.K. (2010). Serial changes in diffusion tensor imaging metrics of corpus callosum in moderate traumatic brain injury patients and their correlation with neuropsychometric tests: A 2-year follow-up study. Journal of Head Trauma Rehabilitation, 25, 31-42.

Lange, R.T., Iverson, G.L., Brubacher, J.R., Mädler, B., \& Heran, M.K. (2012). Diffusion tensor imaging findings are not strongly associated with postconcussional disorder 2 months following mild traumatic brain injury. Journal of Head Trauma Rehabilitation, 27(3), 188-198.

Lange, R.T., Panenka, W.J., Shewchuk, J.R., Heran, M.K.S., Brubacher, J.R., Bioux, S., ... Iverson, G.L. (2015). Diffusion tensor imaging findings and postconcussion symptom reporting six weeks following mild traumatic brain injury. Archives of Clinical Neuropsychology, 30(1), 7-25.

Levin, H.S., Wilde, E., Troyanskaya, M., Petersen, N.J., Scheibel, R., Newsome, M., ... Li, X. (2010). Diffusion tensor imaging of mild to moderate blast-related traumatic brain injury and its sequelae. Journal of Neurotrauma, 27, 683-694.

Ling, J.M., Pena, A., Yeo, R.A., Merideth, F.L., Klimaj, S., Gasparovic, C., \& Mayer, A.R. (2012). Biomarkers of increased diffusion anisotropy in semi-acute mild traumatic brain injury: A longitudinal perspective. Brain, 135, 1281-1292.

Lipton, M.L., Gellella, E., Lo, C., Gold, T., Ardekani, B.A., Shifteh, K., ... Branch, C.A. (2008). Multifocal white matter ultrastructural abnormalities in mild traumatic brain injury with cognitive disability: A voxel-wise analysis of diffusion tensor imaging. Journal of Neurotrauma, 25, 1335-1342.

Lipton, M.L., Gulko, E., Zimmerman, M.E., Friedman, B.W., Kim, M., Gellella, E., ... Branch, C.A. (2009). Diffusion-tensor imaging implicates prefrontal axonal injury in executive function impairment following very mild traumatic brain injury. Radiology, 252, 816-824.

Lipton, M.L., Kim, N., Park, Y.K., Hulkower, M.B., Gardin, T.M., Shifteh, K., ... Branch, C.A. (2012). Robust detection of traumatic axonal injury in individual mild traumatic brain injury patients: Intersubject variation, change over time and bidirectional changes in anisotropy. Brain Imaging and Behavior, 6, 329-342.

Little, D.M., Kraus, M.F., Joseph, J., Geary, E.K., Susmaras, T., Zhou, X.J., Pliskin, N., \& Gorelick, P.B. (2010). Thalamic integrity underlies executive dysfunction in traumatic brain injury. Neurology, 74, 558-564.

Ljungqvist, J., Nilsson, D., Ljungberg, M., Sörbo, A., Esbjörnsson, E., Eriksson-Ritzén, C., \& Skoglund, T. (2011). Longitudinal study of the diffusion tensor imaging properties of the corpus callosum in acute and chronic diffuse axonal injury. Brain Injury, 25, 370-378.

Lo, C., Shifteh, K., Gold, T., Bello, J.A., \& Lipton, M.L. (2009). Diffusion tensor imaging abnormalities in patients with mild traumatic brain injury and neurocognitive impairment. Journal of Computer Assisted Tomography, 33(2), 293-297.

Mac Donald, C.L., Adam, O.R., Johnson, A.M., Nelson, E.C., Werner, N.J., Rivet, D.J., \& Brody, D.L. (2015). Acute posttraumatic stress symptoms and age predict outcome in military blast concussion. Brain, 138(5), 1314-1326.
Mac Donald, C.L., Dikranian, K., Song, S.K., Bayly, P.V., Holtzman, D.M., \& Brody, D.L. (2007). Detection of traumatic axonal injury with diffusion tensor imaging in a mouse model of traumatic brain injury. Experimental Neurology, 205(1), 116-131.

Mac Donald, C.L., Johnson, A.M., Cooper, D., Nelson, E.C., Werner, N.J., Shimony, J.S., ... Brody, D.L. (2011). Detection of blast-related traumatic brain injury in U.S. military personnel. New England Journal of Medicine, 364, 2091-2100.

Marmarou, A., Signoretti, S., Fatouros, P.P., Portella, G., Aygok, G.A., \& Bullock, M.R. (2006). Predominance of cellular edema in traumatic brain swelling in patients with severe head injuries. Journal of Neurosurgery, 104, 720-730.

Marquez de la Plata, C., Garces, J., Kojori, E., Grinnan, J., Krishnan, K., Pidikiti, R., ... Diaz-Arrastia, R. (2011). Deficits in functional connectivity of hippocampal and frontal lobe circuits after traumatic axonal injury. Archives of Neurology, 68, 74-84.

Matsukawa, H., Shinoda, M., Fujii, M., Takahashi, O., Yamamoto, D., Murakata, A., \& Ishikawa, R. (2011). Genu of corpus callosum in diffuse axonal injury induces a worse 1-year outcome in patients with traumatic brain injury. Acta Neurochirurgica, 153(8), 1687-1694.

Matthews, S.C., Strigo, I.A., Simmons, A.N., O’Connell, R.M., Reinhardt, L.E., \& Moseley, S.A. (2011). A multimodal imaging study in US veterans of Operations Iraqi and Enduring Freedom with and without major depression after blast-related concussion. Neuroimage, 54, S69-S75.

Mayer, A.R., Ling, J., Mannell, M.V., Gasparovic, C., Phillips, J.P., Doezema, D., ... Yeo, R.A. (2010). A prospective diffusion tensor imaging study in mild traumatic brain injury. Neurology, 74, 643-650.

Mayer, A.R., Mannell, M., Ling, J., Gasparovic, C., \& Yeo, R. (2011). Functional connectivity in mild traumatic brain injury. Human Brain Mapping, 32, 1825-1835.

McCrea, M., Iverson, G.L., McAllister, T.W., Hammeke, T.A., Powell, M.R., Barr, W.B., \& Kelly, J.P. (2009). An integrated review of recovery after mild traumatic brain injury (MTBI): Implications for clinical management. The Clinical Neuropsychologist, 23(8), 1368-1390.

McKee, A.C., Stein, T.D., Nowinski, C.J., Stern, R.A., Daneshvar, D.H., Alvarez, V.E., ... Baugh, C.M. (2013). The spectrum of disease in chronic traumatic encephalopathy. Brain, 136(1), 43-64.

Messé, A., Caplain, S., Paradot, G., Garrigue, D., Mineo, J.-F., Soto Ares, G., ... Lehéricy, S. (2011). Diffusion tensor imaging and white matter lesions at the subacute stage in mild traumatic brain injury with persistent neurobehavioral impairment. Human Brain Mapping, 32, 999-1011.

Messé, A., Caplain, S., Pélégrini-Issac, M., Blancho, S., Lévy, R., Aghakhani, N., ... Lehéricy, S. (2013). Specific and evolving resting-state network alterations in post-concussion syndrome following mild traumatic brain injury. PLoS One, 8, 1-10.

Messé, A., Caplain, S., Pélégrini-Issac, M., Blancho, S., Montreuil, M., Lévy, R., ... Benali, H. (2012). Structural integrity and postconcussion syndrome in mild traumatic brain injury patients. Brain Imaging and Behavior, 6, 283-292.

Meythaler, J.M., Peduzzi, J.D., Eleftheriou, E., \& Novack, T.A. (2001). Current concepts: Diffuse axonal injury-associated traumatic brain injury. Archives of Physical Medicine and Rehabilitation, 82, 1461-1471.

Miles, L., Grossman, R.I., Johnson, G., Babb, J.S., Diller, L., \& Inglese, M. (2008). Short-term DTI predictors of cognitive dysfunction in mild traumatic brain injury. Brain Injury, 22(2), 115-122. 
Miller, B.T., \& D'Esposito, M. (2005). Searching for "the top" in top-down control. Neuron, 48(4), 535-538.

Moen, K.G., Håberg, A.K., Skandsen, T., Finnanger, T.G., \& Vik, A. (2014). A longitudinal magnetic resonance imaging study of the apparent diffusion coefficient values in corpus callosum during the first year after traumatic brain injury. Journal of Neurotrauma, 31, 56-63.

Morey, R.A., Haswell, C.C., Selgrade, E.S., Massoglia, D., Liu, C., Weiner, J., ... McCarthy, G. (2013). Effects of chronic mild traumatic brain injury on white matter integrity in Iraq and Afghanistan war veterans: Effects of TBI on white matter integrity. Human Brain Mapping, 34, 2986-2999.

Nakamura, T., Hillary, F., \& Biswal, B. (2009). Resting network plasticity following brain injury. PLoS One, 4, 1-9.

Nakayama, N. (2006). Evidence for white matter disruption in traumatic brain injury without macroscopic lesions. Journal of Neurology, Neurosurgery, \& Psychiatry, 77, 850-855.

Newcombe, V.F., Correia, M.M., Ledig, C., Abate, M.G., Outtrim, J.G., Chatfield, D., ... Pickard, J.D. (2015). Dynamic changes in white matter abnormalities correlate with late improvement and deterioration following TBI: A diffusion tensor imaging study. Neurorehabilitation and Neural Repair. doi: 10.1177/154596 8315584004.

Newcombe, V.F.J., Williams, G.B., Nortje, J., Bradley, P.G., Harding, S.G., Smielewski, P., ... Menon, D.K. (2007). Analysis of acute traumatic axonal injury using diffusion tensor imaging. British Journal of Neurosurgery, 21, 340-348.

Niogi, S.N., Mukherjee, P., Ghajar, J., Johnson, C.E., Kolster, R., Lee, H., ... McCandliss, B.D. (2008). Structural dissociation of attentional control and memory in adults with and without mild traumatic brain injury. Brain, 131, 3209-3221.

Ommaya, A.K., \& Gennarelli, T.A. (1974). Cerebral concussion and traumatic unconsciousness. Correlation of experimental and clinical observations of blunt head injuries. Brain, 97, 633-654.

Pal, D., Gupta, R.K., Agarwal, S., Yadav, A., Ojha, B.K., Awasthi, A., ... Narayana, P.A. (2012). Diffusion tensor tractography indices in patients with frontal lobe injury and its correlation with neuropsychological tests. Clinical Neurology and Neurosurgery, 114, 564-571.

Palacios, E., Sala-Llonch, R., Junque, C., Roig, T., Tormos, J., Bargallo, N., \& Vendrell, P. (2012). White matter integrity related to functional working memory networks in traumatic brain injury. Neurology, 78, 852-860.

Palacios, E., Sala-Llonch, R., Junque, C., Roig, T., Tormos, J., Bargallo, N., \& Vendrell, P. (2013). Resting-state functional magnetic resonance imaging activity and connectivity and cognitive outcome in traumatic brain injury. JAMA Neurology, 70, 845-851.

Pandit, A., Expert, P., Lambiotte, R., Bonelle, V., Leech, R., Turkheimer, F., \& Sharp, D. (2013). Traumatic brain injury impairs small-world topology. Neurology, 80, 1826-1833.

Peerless, S.J., \& Rewcastle, N.B. (1967). Shear injuries of the brain. Canadian Medical Association Journal, 96, 577.

Perlbarg, V., Puybasset, L., Tollard, E., Lehéricy, S., Benali, H., \& Galanaud, D. (2009). Relation between brain lesion location and clinical outcome in patients with severe traumatic brain injury: A diffusion tensor imaging study using voxel-based approaches. Human Brain Mapping, 30, 3924-3933.

Petrie, E.C., Cross, D.J., Yarnykh, V.L., Richards, T., Martin, N.M., Pagulayan, K., ... Peskind, E.R. (2014). Neuroimaging, behavioral, and psychological sequelae of repetitive combined blast/impact mild traumatic brain injury in Iraq and Afghanistan War Veterans. Journal of Neurotrauma, 31, 425-436.
Povlishock, J.T., Becker, D.P., Cheng, C.L.Y., \& Vaughan, G.W. (1983). Axonal change in minor head injury. Journal of Neuropathology \& Experimental Neurology, 42(3), 225-242.

Povlishock, J.T., \& Christman, C.W. (1995). The pathobiology of traumatically induced axonal injury in animals and humans: A review of current thoughts. Journal of Neurotrauma, 12, 555-564.

Reinarz, S.J., Coffman, C.E., Smoker, W.R., \& Godersky, J.C. (1988). MR imaging of the corpus callosum: Normal and pathologic findings and correlation with CT. AJNR: American Journal of Neuroradiology, 9, 649-656.

Ruff, R.L., Riechers, R.G., Wang, X.-F., Piero, T., \& Ruff, S.S. (2012). A case-control study examining whether neurological deficits and PTSD in combat veterans are related to episodes of mild TBI. BMJ Open, 2, 1-12.

Ruppin, E., \& Reggia, J.. (1995). Patterns of functional damage in neural network models of associative memory. Neural Computation, 7, 1105-1127.

Ryu, J., Horkayne-Szakaly, I., Xu, L., Pletnikova, O., Leri, F., Eberhart, C., ... Koliatsos, V.E. (2014). The problem of axonal injury in the brains of veterans with histories of blast exposure. Acta Neuropathologica Communications, 2, 1-14.

Salmond, C.H., Menon, D.K., Chatfield, D.A., Williams, G.B., Pena, A., Sahakian, B.J., \& Pickard, J.D. (2006). Diffusion tensor imaging in chronic head injury survivors: Correlations with learning and memory indices. Neuroimage, 29, 117-124.

Sharp, D., Beckmann, C., Greenwood, R., Kinnunen, K., Bonelle, V., De Boissezon, X., ... Leech, R. (2011). Default mode network functional and structural connectivity after traumatic brain injury. Brain, 134, 2233-2247.

Sharp, D., Scott, G., \& Leech, R. (2014). Network dysfunction after traumatic brain injury. Nature Reviews Neurology, 10, 156-166.

Shenton, M.E., Hamoda, H.M., Schneiderman, J.S., Bouix, S., Pasternak, O., Rathi, Y., ... Zafonte, R. (2012). A review of magnetic resonance imaging and diffusion tensor imaging findings in mild traumatic brain injury. Brain Imaging and Behavior, 6, 137-192.

Shumskaya, E., Andriessen, T., Norris, D., \& Vos, P. (2012). Abnormal whole-brain functional networks in homogeneous acute mild traumatic brain injury. Neurology, 79, 175-182.

Sidaros, A., Engberg, A.W., Sidaros, K., Liptrot, M.G., Herning, M., Petersen, P., ... Rostrup, E. (2008). Diffusion tensor imaging during recovery from severe traumatic brain injury and relation to clinical outcome: A longitudinal study. Brain, 131, 559-572.

Skoglund, T.S., Nilsson, D., Ljungberg, M., Jönsson, L., \& Rydenhag, B. (2008). Long-term follow-up of a patient with traumatic brain injury using diffusion tensor imaging. Acta Radiologica, 49, 98-100.

Smith, D.H., Meaney, D.F., \& Shull, W.H. (2003). Diffuse axonal injury in head trauma. Journal of Head Trauma Rehabilitation, 18, 307-316.

Smith, S.M. (2012). The future of FMRI connectivity. Neuroimage, $62,1257-1266$.

Smits, M., Houston, G.C., Dippel, D.W.J., Wielopolski, P.A., Vernooij, M.W., Koudstaal, P.J., ... van Der Lugt, A. (2011). Microstructural brain injury in post-concussion syndrome after minor head injury. Neuroradiology, 53(8), 553-563.

Sorg, S.F., Delano-Wood, L., Luc, N., Schiehser, D.M., Hanson, K.L., Nation, D.A., ... Meloy, M. (2013). White matter integrity in veterans with mild traumatic brain injury: Associations with executive function and loss of consciousness. The Journal of Head Trauma Rehabilitation, 29(1), 21-32. 
Sours, C., Zhuo, J., Janowich, J., Aarabi, B., Shanmuganathan, K., \& Gullapalli, R. (2013). Default mode network interference in mild traumatic brain injury - A pilot resting state study. Brain Research, 1537, 201-215.

Sporns, O. (2014). Contributions and challenges for network models in cognitive neuroscience. Nature Neuroscience, 17, 652-660.

Stevens, M., Lovejoy, D., Kim, J., Oakes, H., Kureshi, I., \& Witt, S. (2012). Multiple resting state network functional connectivity abnormalities in mild traumatic brain injury. Brain Imaging and Behavior, 6, 293-318.

Strich, S.J. (1956). Diffuse degeneration of the cerebral white matter in severe dementia following head injury. Journal of Neurology, Neurosurgery, and Psychiatry, 19, 163.

Suskauer, S.J., \& Huisman, T.A. (2009). Neuroimaging in pediatric traumatic brain injury: Current and future predictors of functional outcome. Developmental Disabilities Research Reviews, 15(2), 117-123.

Taber, K.H., \& Hurley, R.A. (2013). Update on mild traumatic brain injury: Neuropathology and structural imaging. The Journal of Neuropsychiatry and Clinical Neurosciences, 25(1), 1-5.

Taber, K.H., Hurley, R.A., Haswell, C.C., Rowland, J.A., Hurt, S.D., Lamar, C.D., \& Morey, R.A. (2015). White matter compromise in veterans exposed to primary blast forces. Journal of Head Trauma Rehabilitation, 30(1), E15-E25.

Tang, L., Ge, Y., Sodickson, D., Miles, L., Zhou, Y., Reaume, J., ... Grossman, R. (2011). Thalamic resting-state functional networks: Disruption in patients with mild traumatic brain injury. Radiology, 260, 831-840

Tate, D.F., \& Bigler, E.D. (2000). Fornix and hippocampal atrophy in traumatic brain injury. Learning \& Memory, 7(6), 442-446.

Tomaszczyk, J.C., Green, N.L., Frasca, D., Colella, B., Turner, G.R., Christensen, B.K., \& Green, R.E.A. (2014). Negative neuroplasticity in chronic traumatic brain injury and implications for neurorehabilitation. Neuropsychology Review, 24, 409-427.

Tournier, J.D., Mori, S., \& Leemans, A. (2011). Diffusion tensor imaging and beyond. Magnetic Resonance in Medicine, 65(6), 1532-1556.

van den Heuvel, M.P., \& Sporns, O. (2011). Rich-club organization of the human connectome. The Journal of Neuroscience, 31(44), 15775-15786.

Venkatesan, U., Dennis, N., \& Hillary, F. (2015). Chronology and chronicity of altered resting-state functional connectivity after traumatic brain injury. Journal of Neurotrauma, 32, 252-264.

Voss, H.U., Uluç, A.M., Dyke, J.P., Watts, R., Kobylarz, E.J., McCandliss, B.D., ... Vallabhajosula, S. (2006). Possible axonal regrowth in late recovery from the minimally conscious state. Journal of Clinical Investigation, 116(7), 2005.
Wäljas, M., Lange, R.T., Hakulinen, U., Huhtala, H., Dastidar, P., Hartikainen, K., ... Iverson, G.L. (2014). Biopsychosocial outcome after uncomplicated mild traumatic brain injury. Journal of Neurotrauma, 31(1), 108-124.

Wang, J.Y., Bakhadirov, K., Abdi, H., Devous, M.D., Marquez de la Plata, C.D., Moore, C., ... Diaz-Arrastia, R. (2011). Longitudinal changes of structural connectivity in traumatic axonal injury. Neurology, 77, 818-826.

Warden, D.L., French, L.M., Shupenko, L., Fargus, J., Riedy, G., Erickson, M.E., ... Moore, D.F. (2009). Case report of a soldier with primary blast brain injury. Neuroimage, 47, T152-T153.

Watts, R., Thomas, A., Filippi, C.G., Nickerson, J.P., \& Freeman, K. (2014). Potholes and molehills: Bias in the diagnostic performance of diffusion-tensor imaging in concussion. Radiology, 272(1), 217-223.

Wilde, E.A., Hunter, J.V., \& Bigler, E.D. (2012). Neuroimaging in neurorehabilitation. NeuroRehabilitation, 31, 223-226.

Xu, J., Rasmussen, I.-A., Lagopoulos, J., \& Håberg, A. (2007). Diffuse axonal injury in severe traumatic brain injury visualized using high-resolution diffusion tensor imaging. Journal of Neurotrauma, 24(5), 753-765.

Yeh, P.H., Wang, B., Oakes, T.R., French, L.M., Pan, H., Graner, J., ... Riedy, G. (2014). Postconcussional disorder and PTSD symptoms of military-related traumatic brain injury associated with compromised neurocircuitry: Neurocircuitry in military TBI and clinical symptoms. Human Brain Mapping, 35, 2652-2673.

Yeo, B., Krienen, F., Sepulcre, J., Sabuncu, M., Lashkari, D., Hollinshead, M., ... Buckner, R. (2011). The organization of the human cerebral cortex estimated by intrinsic functional connectivity. Journal of Neurophysiology, 106, 1125-1165.

Yuh, E.L., Cooper, S.R., Mukherjee, P., Yue, J.K., Lingsma, H.F., Gordon, W.A., ... Vassar, M.J. (2014). Diffusion tensor imaging for outcome prediction in mild traumatic brain injury: A TRACKTBI study. Journal of Neurotrauma, 31(17), 1457-1477.

Zhang, S., \& Li, C.S. (2012). Functional connectivity mapping of the human precuneus by resting state fMRI. Neuroimage, 59, 3548-3562.

Zhou, Y., Lui, Y., Zuo, X., Milham, M., Reaume, J., Grossman, R., $\& \mathrm{Ge}, \mathrm{Y}$. (2014). Characterization of thalamo-cortical association using amplitude and connectivity of functional MRI in mild traumatic brain injury. Journal of Magnetic Resonance Imaging, 39, 1558-1568.

Zhou, Y., Milham, M., Lui, Y., Miles, L., Reaume, J., Sodickson, D., ... Ge, Y. (2012). Default-mode network disruption in mild traumatic brain injury. Radiology, 265, 882-892. 